\title{
Three Galactic globular cluster candidates ${ }^{\star}$
}

\author{
C. Moni Bidin ${ }^{1}$, F. Mauro ${ }^{1}$, D. Geisler ${ }^{1}$, D. Minniti ${ }^{2}$, M. Catelan² ${ }^{2}$, M. Hempel $^{2}$, E. Valenti ${ }^{3}$, A. A. R. Valcarce ${ }^{2,5}$, \\ J. Alonso-García ${ }^{2}$, J. Borissova ${ }^{6}$, G. Carraro ${ }^{4}$, P. Lucas ${ }^{7}$, A.-N. Chené ${ }^{1,6}$, M. Zoccali ${ }^{2}$, and R. G. Kurtev ${ }^{6}$ \\ ${ }^{1}$ Departamento de Astronomía, Universidad de Concepción, Casilla 160-C, Concepción, Chile \\ e-mail: cmbidin@astro-udec.cl \\ 2 Departamento de Astronomía y Astrofísica, Pontificia Universidad Católica de Chile, Casilla 306, Santiago, Chile \\ 3 European Southern Observatory, Karl-Schwarzschild-Straße 2, 85748 Garching bei München, Germany \\ ${ }^{4}$ European Southern Observatory, Avda Alonso de Cordova, 3107, Casilla 19001, Santiago, Chile \\ 5 Departamento de Física, Universidade Federal do Rio Grande do Norte, 59072-970 Natal, RN, Brazil \\ ${ }^{6}$ Departamento de Física y Astronomía, Facultad de Ciencias, Universidad de Valparaíso, Av. Gran Bretaña 1111, Valparaíso, Chile \\ 7 Centre for Astrophysics Research, Science and Technology Research Institute, University of Hertfordshire, \\ Hatfield AL10 9AB, UK
}

Received 16 June 2011 / Accepted 30 August 2011

\section{ABSTRACT}

\begin{abstract}
Context. The census of Galactic globular clusters (GCs) is still incomplete, and about ten new objects are supposed to await discovery, hidden behind the crowded and reddened regions of the Galactic bulge and disk.

Aims. We investigated the nature of three new GC candidates, discovered in the frames collected by the Vista Variables in the Via Lactea (VVV) near-infrared survey. They will be called VVV CL002, VVV CL003, and VVV CL004.

Methods. We studied the results of point-spread-function near-infrared photometry from VVV data for the three objects and their surrounding fields, the proper motion information available in the literature and, when possible, we derived the cluster parameters by means of calibrated indices measured on the color-magnitude diagrams.

Results. The evidence shows that VVV CL002 is a newly discovered, small, moderately metal-rich $([\mathrm{Fe} / \mathrm{H}] \approx-0.4) \mathrm{Galactic}$ GC. It is located at a Galactocentric distance of $0.7 \pm 0.9 \mathrm{kpc}$, and it could be one of the nearest GC to the Galactic center. Its characteristics are more similar to those of low-mass, Palomar-like GCs than to more classical, old, and massive bulge GCs. VVV CL003 is the first star cluster discovered in the Galactic disk on the opposite side of the center with respect to the Sun, at a Galactocentric distance of $\sim 5 \mathrm{kpc}$. Its high metallicity $([\mathrm{Fe} / \mathrm{H}] \approx-0.1)$ and location point to an open cluster, but a GC cannot be excluded. VVV CL004, on the contrary, is most probably only a random clump of field stars, as indicated by both its low statistical significance and by the impossibility to distinguish its stars from the surrounding field population.

Conclusions. We claim the detection of i) a new Galactic GC, deriving an estimate of its basic parameters; ii) a stellar aggregate, probably an open cluster, in the disk directly beyond the Galactic center; and iii) an overdensity of stars, most probably an asterism.
\end{abstract}

Key words. surveys - globular clusters: individual: VVV CL002 - globular clusters: individual: VVV CL003 globular clusters: general

\section{Introduction}

The census of Galactic globular clusters (GCs) is still incomplete, because distant objects can easily hide behind the very crowded and highly reddened stellar fields in the direction of the Galactic bulge and disk. Indeed, Ivanov et al. (2005) estimated that there should be $10 \pm 3$ undiscovered GCs hiding in the inner Milky Way. The advent of the new generation of extensive surveys such as SDSS (Abazajian et al. 2009), 2MASS (Skrutskie et al. 2006), and GLIMPSE (Benjamin et al. 2003) permitted the detection of several new Galactic GCs. The December 2010 compilation of the Harris (1996) catalog included seven new GCs not present in the February 2003 version, but seven more objects have been proposed in the last years: SDSSJ1257+3419 (Sakamoto \& Hasegawa 2006), FSR 584 (Bica et al. 2007), FSR 1767 (Bonatto et al. 2007), FSR 190 (Froebrich et al. 2008a), Pfleiderer 2 (Ortolani et al. 2009), VVV CL001 (Minniti et al. 2011), and Mercer 5 (Longmore et al. 2011). The family of

* Based on observations gathered with ESO-VISTA telescope (proposal ID 172.B-2002).
Galactic GCs is thus steadily increasing, although the true nature of the GC candidates is not always easy to unveil, and some objects are still debated (see, for example, Froebrich et al. 2008b).

Globular clusters are old objects, whose age ranges from about half to nearly one Hubble time. Their spatial distribution and physical/chemical properties thus bear information about the Galactic formation and evolution processes. However, they continuously loose mass during their entire lifetime because of both external processes such as Galactic tidal stress, and internal ones such as stellar evaporation (Meylan \& Heggie 1997; Gnedin \& Ostriker 1997). The identification and the study of low-mass GCs in the Galactic bulge can therefore provide new information about both its formation and chemical enrichment history, and the tidal forces governing its dense environment.

In this paper we analyze three new cluster candidates discovered in the VISTA Variables in the Via Lactea (VVV) Public Survey, which is gathering near-IR data of the Galactic bulge and inner disk (Minniti et al. 2010; Saito et al. 2010; Catelan et al. 2011). This survey is scanning the central regions of the Milky Way with unprecedented depth and extension, thus generating the ideal database to search for missing GCs in the inner Galaxy. 
Following the nomenclature introduced by Minniti et al. (2011) and Borissova et al. (2011), the objects under study here will be called VVV CL002, VVV CL003, and VVV CL004.

\section{Photometric data}

\subsection{The cluster candidates}

VVV CL002, VVV CL003, and VVV CL004 were identified as previously unknown stellar overdensities by visual inspection of the three-band $\left(J, H, K_{\mathrm{s}}\right)$, color composite frames produced by the VVV survey. Table 1 reports for each object the corresponding VVV fields and the J2000 coordinates of their center, as determined in Sect. 3.1. Figure 1 shows the three objects in the $K_{\mathrm{s}}$-band stacked tiles downloaded from the Vista Science Archive website ${ }^{1}$ (VSA). VVV CL002 and VVV CL003 look like loose and low-mass, Palomar-like GCs, although VVV CL003 is less clear because it is embedded in a very crowded stellar field. VVV CL004, on the contrary, does not show a clear concentration of faint stars, but only a local overdensity of a few bright objects.

\subsection{Data reduction and photometry}

After the discovery, we retrieved from VSA the stacked images of the individual $2048 \times 2048$ pixel exposures containing the three candidate clusters. The quantity of frames used in each band is indicated in Table 1. The effective exposure time of the $H$ - and $K_{\mathrm{s}}$-band images was $8 \mathrm{~s}$, while it was $24 \mathrm{~s}$ in $J$. The frames in the $Y$ and $Z$ bands, included in the observation plan of the VVV survey, were not available at the time of the investigation. The data were acquired on 2010 April 7 to 14, with the VIRCAM camera mounted on the VISTA $4 \mathrm{~m}$ telescope at the Paranal Observatory (Emerson \& Sutherland 2010), and reduced at the Cambridge Astronomical Survey Unit (CASU) ${ }^{2}$ with the VIRCAM pipeline v1.0 (Irwin et al. 2004). During the observations the weather conditions fell within the survey's constraints for seeing, airmass, and Moon distance (Minniti et al. 2010), and the quality of the data was satisfactory.

Stellar photometry was performed with the DAOPHOT II and ALLFRAME codes (Stetson 1994). After the construction of the point-spread function (PSF) for each frame and an initial profile-fitting photometry, all images of the same object were matched to create a combined frame, where the master list of star-like sources was created. A PSF fitting of this master list was then performed on each single exposure. The instrumental magnitudes were transformed into the 2MASS photometric system by means of the 2MASS stars found in each field. For each frame the 2MASS sources were first identified in our catalog, then the list was cleaned of objects that in our photometry had a detected source nearer than 2 '. 2 and fainter by less than 3.88 mag. We thus excluded the stars whose 2MASS aperture photometry could have been contaminated by nearby undetected sources by more than $0.03 \mathrm{mag}$. We also limited the selection of calibration stars in the range $J_{2 \mathrm{MASS}}=11.5-13, H_{2 \mathrm{MASS}}=10-11$, and $K_{2 \mathrm{MASS}, \mathrm{s}}=9.5-11$, to avoid stars heavily saturated in VVV data and the fainter end of the 2MASS survey, where systematic errors can be significant (see below). Between 70 and 490 stars met all criteria in each frame. The calibration equations were in the form $m_{2 \mathrm{MASS}}-m=A+B \times\left(J-K_{\mathrm{S}}\right)_{2 \mathrm{MASS}}$, where $m_{2 \mathrm{MASS}}$ and $m$ are the 2MASS and instrumental magnitudes, respectively, and

\footnotetext{
${ }^{1}$ http://horus.roe.ac.uk/vsa/

2 http://casu.ast.cam.ac.uk/
}

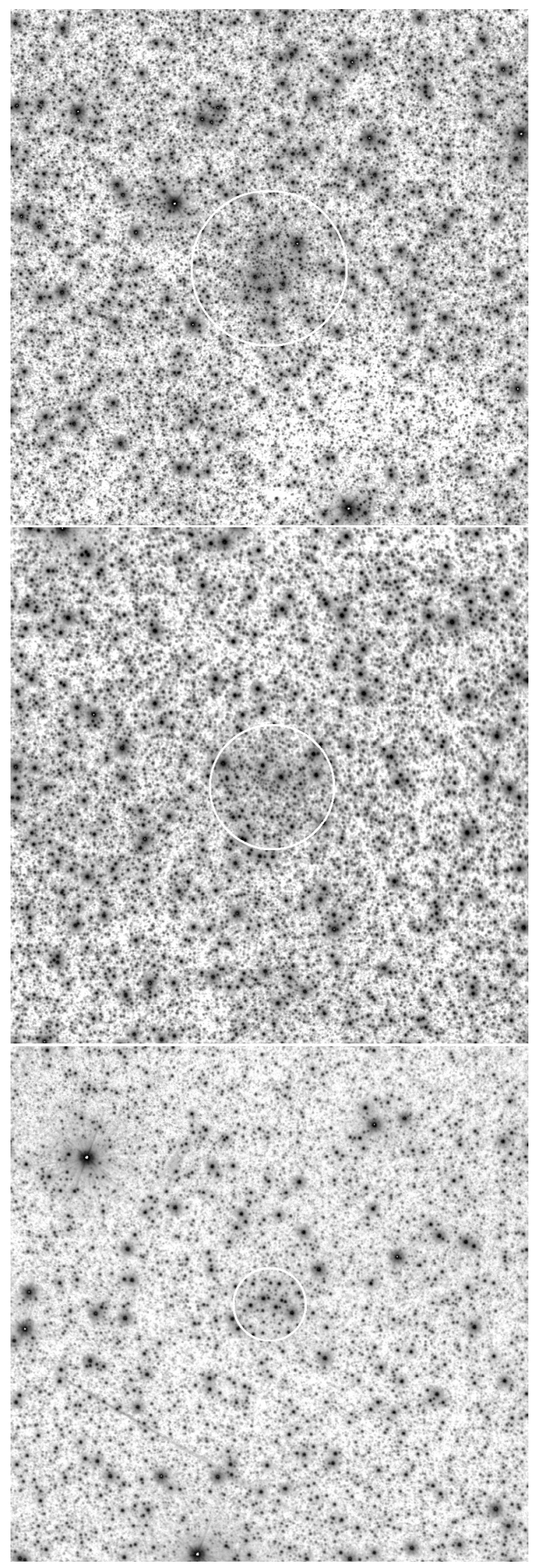

Fig. 1. $5^{\prime} \times 5^{\prime}$ logarithmic gray-scale $K_{\mathrm{s}}$-band images from the VVV archive, centered on the three candidate clusters (from upper to lower panel: VVV CL002, VVV CL003, and VVV CL004). North is up and East is to the right. The white circles, centered on the derived cluster center, indicate the half-light radius estimated for each object. 
C. Moni Bidin et al.: Three Galactic globular cluster candidates

Table 1. Coordinates of the center of the three GC candidates, and the VVV data used in the present investigation.

\begin{tabular}{lccccccc}
\hline \hline ID & $\begin{array}{c}\text { RA } \\
\text { hh:mm:ss }\end{array}$ & $\begin{array}{c}\text { Dec } \\
\text { deg:mm:ss }\end{array}$ & $\begin{array}{c}l \\
\text { deg }\end{array}$ & $\begin{array}{c}b \\
\text { deg }\end{array}$ & VVV field & Frames & Classification \\
\hline VVV CL002 & $17: 41: 06.30$ & $-28: 50: 42.3$ & 359.5586 & 0.8888 & b347 & $2 \times J, 2 \times H, 4 \times K_{\mathrm{s}}$ & GC \\
VVV CL003 & $17: 38: 54.56$ & $-29: 54: 25.3$ & 358.4047 & 0.7298 & b346 & $2 \times J, 2 \times H, 4 \times K_{\mathrm{s}}$ & OC? \\
VVV CL004 & $17: 54: 31.91$ & $-22: 13: 37.8$ & 6.7900 & 1.7179 & b352 & $4 \times J, 4 \times H, 4 \times K_{\mathrm{s}}$ & NC \\
\hline
\end{tabular}

Notes. The classification $(\mathrm{GC}=$ globular cluster, $\mathrm{OC}=$ open cluster, $\mathrm{NC}=$ not a cluster) resulting from our investigation is given in the last column .

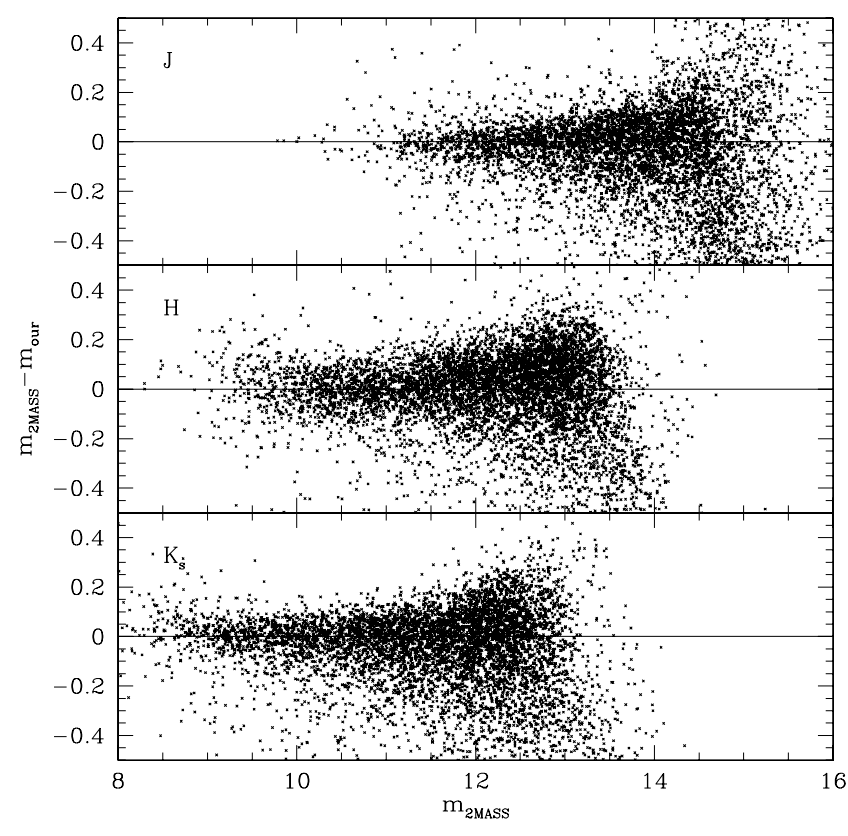

Fig. 2. Magnitude difference between 2 MASS and our photometry for all the stars in common as a function of 2MASS magnitude in the stellar field of VVV CL002. From top to bottom: $J, H$, and $K_{\mathrm{s}}$ magnitudes.

$A$ and $B$ are constants. The color term is in general negligible, being on the order of few hundredths of magnitude. In Table 2 we give the average coefficients $A$ and $B$ for each pair of offsets collected within a few minutes of each other, and the rms of the points around the fitted relation. The final calibrated photometry was checked studying the magnitude difference with respect to the 2MASS catalog. These residuals showed no trend with color, but a systematic difference was found for the fainter stars in all bands and fields. As an example, the results of this comparison for VVV CL002 and its stellar field is shown in Fig. 2, but very similar plots were obtained for the other two cluster candidates as well. The numerous and dispersed negative values indicate the 2MASS stars contaminated by undetected sources. The agreement is satisfactory for the brighter stars, but in the faintest $\sim 1.5$ mag the bulk of the distribution curves upward to positive values, indicating that the stars are fainter in the 2MASS catalog than in our photometry. This feature was found identical even when performing PSF photometry of VVV data with the DoPhot code (Schechter et al. 1993), and when comparing the 2MASS magnitudes with the VVV aperture photometry downloaded from VSA, whose catalogs agree well with our results. As an example, in Fig. 3 our $J$ magnitudes in the VVV CL002 stellar field are compared to the corresponding quantity in the VSA catalog. The same data that show a systematic difference when compared to 2MASS (upper panel of Fig. 2) do not reveal

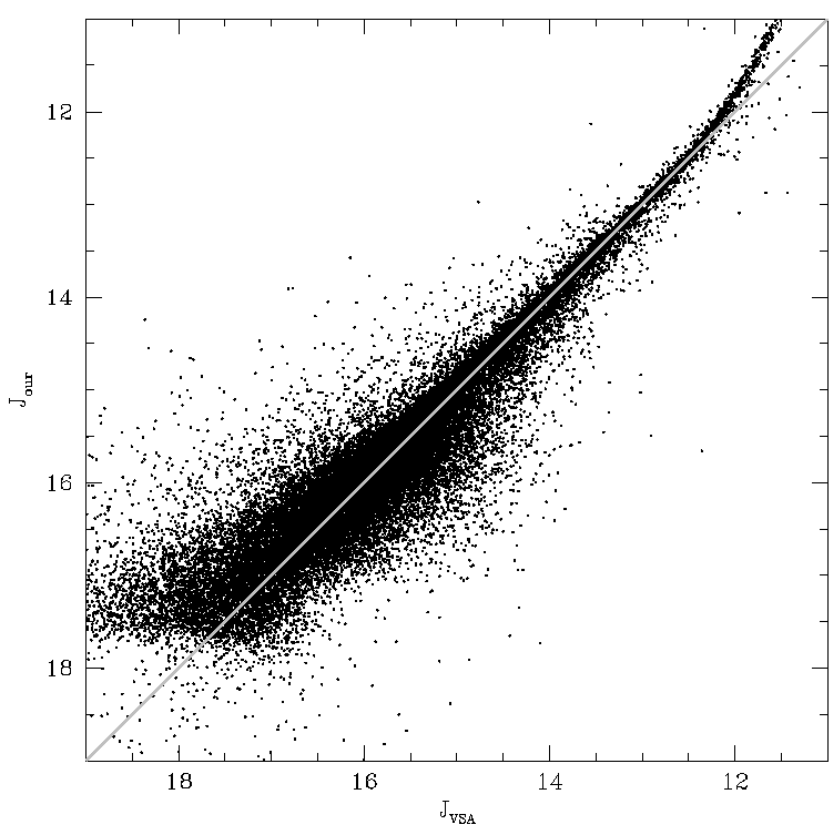

Fig. 3. Comparison between our $J$-band magnitude $\left(J_{\text {our }}\right)$ and the VSA aperture photometry catalog $\left(J_{\mathrm{VSA}}\right)$, for stars in the stellar field of VVV CL002. The gray line indicates the zero-difference between the two photometries $\left(J_{\mathrm{VSA}}=J_{\text {our }}\right)$.

any trend in this plot, apart from an expected mismatch for stars in the saturation regime $(J \leq 12.5)$, where the VSA aperture photometry underestimates the stellar brightness. We conclude that this systematic offset reflects an intrinsic difference between 2MASS and VVV data. We believe it is caused by the relative shallowness of the former and its poorer pixel scale, which leads to an overestimate of the sky background, especially in crowded fields, and consequently to an overly-faint magnitude estimate for faint stars. A more detailed analysis is beyond the scope of this paper, and it will be presented in a future study (Mauro et al. in prep.).

VVV CL003 was found in frames acquired with the chips \#12 and \#16 of the VIRCAM camera. The latter chip is never correctly flat-fielded because of short-timescale variations of its quantum efficiency ${ }^{3}$. We found that the PSF photometry resulting from this chip was unreliable, because of large zero-point variations on the $\mathrm{CCD}$. We finally decided to exclude these frames, and only the exposures collected with chip \#12, listed in Table 1, were used. Unfortunately, the object fell on the edge of this detector, where the photometric results are of lower quality, and the cluster center was only $\sim 19^{\prime \prime}$ from the border. Only half of the surrounding field was therefore available for the analysis,

\footnotetext{
${ }^{3}$ http://casu.ast.cam.ac.uk/surveys-projects/vista/ technical/known-issues
} 
Table 2. Average calibration coefficients for each pair of contiguous offsets.

\begin{tabular}{cccccc}
\hline \hline Date & UT & Filter & $\begin{array}{c}A \\
\text { mag }\end{array}$ & $B$ & $\begin{array}{c}\text { rms } \\
\text { mag }\end{array}$ \\
\hline 2010-04-07 & $7: 17: 28$ & $H$ & $0.17 \pm 0.03$ & $-0.013 \pm 0.011$ & 0.057 \\
$2010-04-07$ & $7: 20: 46$ & $K_{\mathrm{s}}$ & $-0.47 \pm 0.02$ & $-0.060 \pm 0.009$ & 0.061 \\
$2010-04-07$ & $7: 24: 40$ & $J$ & $0.64 \pm 0.02$ & $0.009 \pm 0.008$ & 0.053 \\
$2010-04-14$ & $9: 32: 23$ & $K_{\mathrm{s}}$ & $-0.56 \pm 0.02$ & $-0.028 \pm 0.008$ & 0.052 \\
\hline \multicolumn{7}{c}{ VVV CL003-chip 12 } \\
2010-04-07 & $6: 52: 54$ & $H$ & $0.23 \pm 0.02$ & $-0.014 \pm 0.010$ & 0.053 \\
$2010-04-07$ & $6: 56: 11$ & $K_{\mathrm{s}}$ & $-0.561 \pm 0.016$ & $-0.017 \pm 0.007$ & 0.058 \\
$2010-04-07$ & $7: 01: 05$ & $J$ & $0.55 \pm 0.02$ & $0.039 \pm 0.008$ & 0.041 \\
$2010-04-14$ & $9: 06: 35$ & $K_{\mathrm{s}}$ & $-0.559 \pm 0.014$ & $-0.019 \pm 0.006$ & 0.053 \\
\hline \multicolumn{7}{c}{ VVV CL004-chip 5 } \\
$2010-04-11$ & $5: 41: 06$ & $H$ & $0.278 \pm 0.015$ & $-0.048 \pm 0.010$ & 0.033 \\
$2010-04-11$ & $5: 44: 13$ & $K_{\mathrm{s}}$ & $-0.554 \pm 0.009$ & $-0.025 \pm 0.006$ & 0.036 \\
$2010-04-11$ & $5: 48: 40$ & $J$ & $0.592 \pm 0.011$ & $0.039 \pm 0.008$ & 0.032 \\
\hline \multicolumn{7}{c}{ VVV CL004-chip 9 } \\
$2010-04-11$ & $5: 40: 12$ & $H$ & $0.230 \pm 0.010$ & $-0.014 \pm 0.007$ & 0.028 \\
$2010-04-11$ & $5: 43: 20$ & $K_{\mathrm{s}}$ & $-0.563 \pm 0.010$ & $-0.014 \pm 0.007$ & 0.032 \\
$2010-04-11$ & $5: 47: 07$ & $J$ & $0.622 \pm 0.011$ & $0.020 \pm 0.007$ & 0.030 \\
\hline \multicolumn{7}{c}{}
\end{tabular}

and we estimated by eye that we have studied only about threefourths of the candidate cluster.

\section{Results}

\subsection{Cluster center}

With the final photometry of the three cluster candidates, we revised the location of their centers, first estimated by eye. This calculation was affected by a smooth background of numerous faint field sources that shifted the barycenter of the stellar distribution toward the center of the region selected for the calculation. Cuts in the color-magnitude diagrams did not help, because either the distribution of cluster and field stars was similar (VVV CL002 and VVV CL004, see Fig. 6), or the photometric information was not reliable (VVV CL003, see Sect. 2.2). However, if $\boldsymbol{r}$ is the position of the center of the stellar distribution, and $N$ is the number of stars, we can write

$\boldsymbol{r}_{*} N_{*}=\sum_{i} \boldsymbol{r}_{i}=\boldsymbol{r}_{\mathrm{c}} N_{\mathrm{c}}+\boldsymbol{r}_{\mathrm{f}} N_{\mathrm{f}}$,

where $\boldsymbol{r}_{i}$ is the position of the $i$ th star, and the subscripts *, f, and c refer to all, field, and cluster stars, respectively. After simple algebra, considering that $N_{*}=N_{\mathrm{c}}+N_{\mathrm{f}}$, and that the center of the distribution of field stars $\left(\boldsymbol{r}_{\mathrm{f}}\right)$ coincides with the center of the area included in the calculation $\left(\boldsymbol{r}_{0}\right)$, the previous relation yields

$\boldsymbol{r}_{\mathrm{c}}=\boldsymbol{r}_{0}+\frac{\sum_{i}\left(\boldsymbol{r}_{i}-\boldsymbol{r}_{0}\right)}{N_{*}-N_{\mathrm{f}}}$

This formula was used to find the position of the centers of the three objects. The total number of field stars $\left(N_{\mathrm{f}}\right)$ was estimated from the average stellar density in regions farther than $3^{\prime}$ from the initial by-eye center position. To limit the influence of random fluctuations of the stellar distribution, the calculation was repeated in 360 areas of $3^{\prime}$ radius, whose centers were equally spaced on a circle of 0.5 radius around the initial guess. The results differed by less than $1^{\prime \prime}$ with an rms of only 0.3 , and they were averaged to obtain the final cluster center, given in Table 1.

To cover a larger field for VVV CL003, the stellar counts were performed including the data collected with chip \#16.

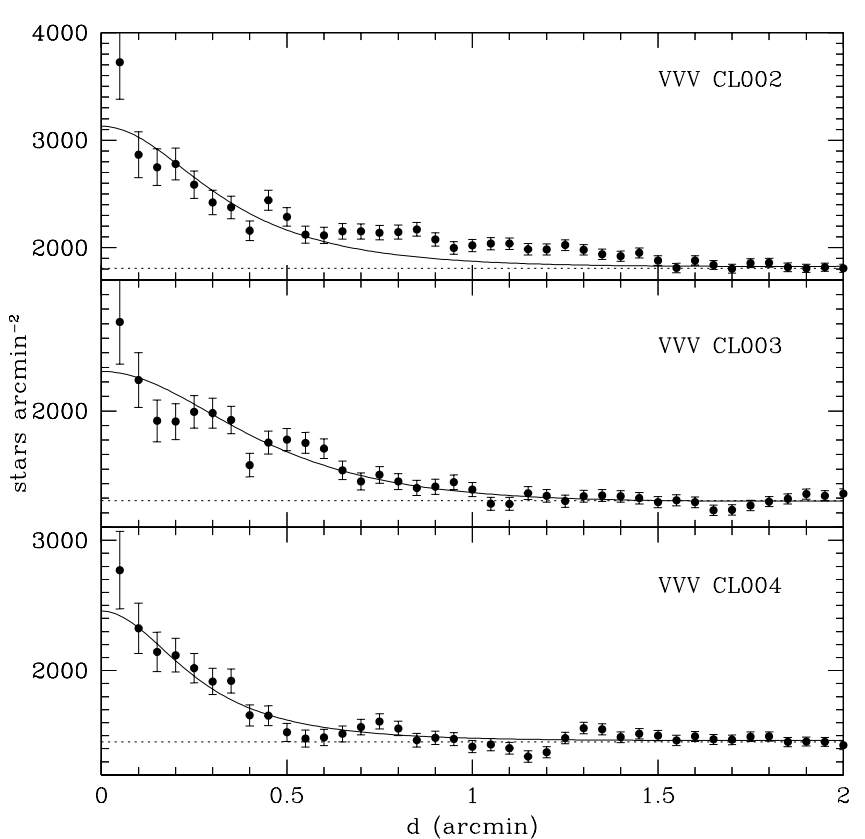

Fig. 4. Radial profile of the stellar surface density for the three cluster candidates. The dotted lines show the estimated field level, while the curve indicates the best-fit King profile.

As already noted, these data are not suitable for photometry though they are still good enough for star count purposes.

\subsection{Significance of the stellar overdensity}

Following Koposov et al. (2007), we can estimate the statistical significance of the stellar overdensities from the number of stars detected in excess to the local background, whose random fluctuations are assumed to be Poissonian. We detected 6965 stars in the inner circle of radius $1^{\prime}$ of VVV CL002, while the field density level can account only for 5744 objects: an overdensity of stars at the $10.9 \sigma$ level is thus present in this area. Analogously, 5193 stars are detected for VVV CL003, against a field expectation of 4313, and the overdensity is significant at the $9 \sigma$ level. The quantity of stars detected in excess of the field expectation within $1^{\prime}$ from the center of VVV CL004 is lower ( 400), and the statistical significance is $\sim 4.5 \sigma$. The 520 square degrees covered by the VVV survey can be divided into $\sim 600000$ circles of radius $1^{\prime}$, therefore the probability of detecting one or more such overdensity in the whole survey that is caused by random field fluctuations is $\sim 98 \%$, and about four similar objects should be expected. Hence, the statistical significance of VVV CL004 is low, casting serious doubts on its nature as a true stellar cluster.

\subsection{Radial density profile}

After the determination of the cluster center, we calculated the stellar density (number of stars per $\operatorname{arcmin}^{2}$ ) in concentric rings between the circles of radius $0.05 \times N$ and $00^{\prime} 05 \times N+0{ }^{\prime} .1$ (with $N=0,1,2,3 \ldots$. . The resulting radial profiles of the stellar density are shown in Fig. 4, where the error bars are given by the Poissonian noise. The field density level, indicated by a dotted line, was assumed as the average value between 1!.8 and $2^{\prime}$ from the center, i.e. beyond the clusters' tidal radii determined below. All three objects show a clustering of stars in the central region. 


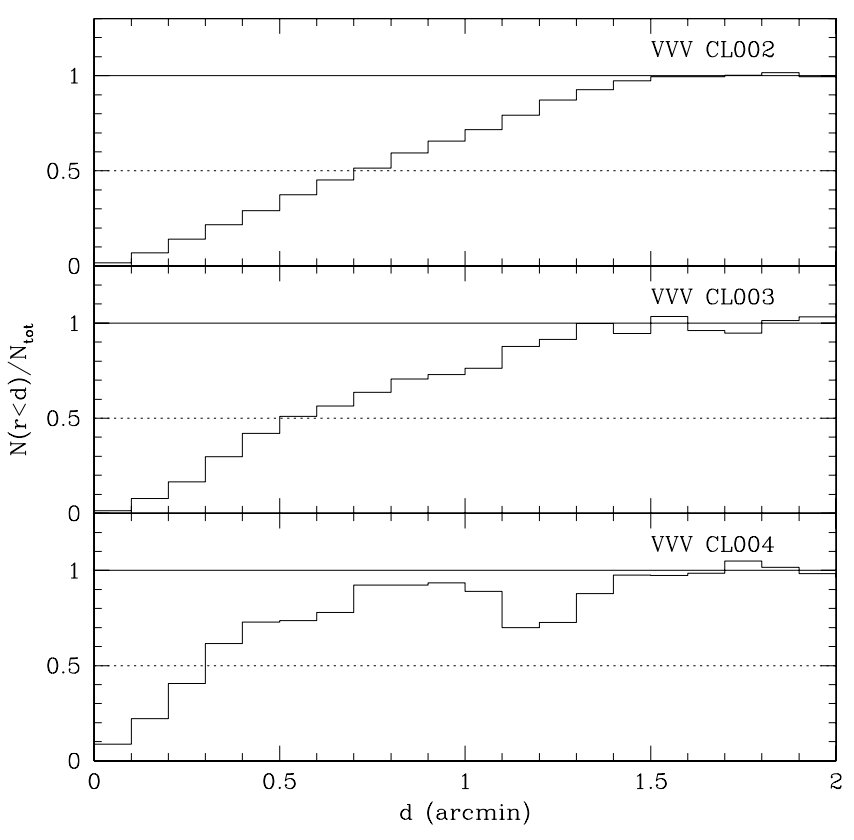

Fig. 5. Cumulative fraction of cluster stars as a function of distance from the center. The field and the half-light levels are indicated with full and dotted lines, respectively.

The innermost bin shows a sudden increase of the density in all cases, but this value is not very reliable because it corresponds to a circle of only 0 ' 1 radius. On the other hand, the possibility of photometric incompleteness in the most crowded region must be taken into account in the following analysis. If the catalogs were incomplete in the inner part of the cluster candidates, the statistical significance of the detected stellar overdensity would be underestimated, and the decontaminated color-magnitude diagrams would be relatively deficient of stars, mainly at fainter magnitudes. However, we detect between 80 and 100 stars in the inner $0{ }^{\prime} 1$ of the three clusters, that is 225 times the area covered by a circle of diameter equal to the FWHM of the PSF ( 0 ' $\left.^{\prime} 8\right)$. This indicates that the three objects are not excessively crowded, and the incompleteness should not be very severe.

The radial profiles of VVV CL002 and VVV CL003 are very similar, smoothly falling off at increasing distance into an extended tail. On the contrary, the density of VVV CL004 drops more abruptly, but the cluster limit is not immediately evident because of strong density fluctuations. All these conclusions are confirmed and better visualized by the radial profiles of the cumulative fraction of stars, presented in Fig. 5. These were calculated counting the stars detected within a given radius, and subtracting the number of expected field stars, estimated from the average density between 1'.8 and $2^{\prime}$ from the center. This range was also used to derive the total number of cluster stars that were used to normalize the curve. The resulting profiles show that VVV CL002 extends out to $\sim 1$ '.6 from the center, and its half-light radius, i.e. the radius containing half of the detected stars, is $r_{\mathrm{h}} \sim 0$ '.75. The apparent size of VVV CL003 is probably smaller because it fades into the field density between 1.4 and 1.'6 from the center, and its half-light radius is $r_{\mathrm{h}} \sim 00^{\prime} 6$. The angular size of VVV CL004 is more uncertain, because the field density is patchy and both the density profile and the cumulative fraction of stars fluctuate strongly. The radius where the density merges into the field continuum is unclear, probably comprised between $1^{\prime}$ and 1!'7, while $r_{\mathrm{h}} \sim 0$ '35. The radial density profile of the three objects was fitted with a King (1966) profile, and the results are overplotted on the observational data in Fig. 4. For VVV CL003 this procedure returned a very good fit of the data, with a tidal radius $r_{\mathrm{t}}=1.8$ and a central concentration $c=\log \left(r_{\mathrm{t}} / r_{\mathrm{c}}\right)=0.56$, where $r_{\mathrm{c}}$ is the core radius. The density profile of VVV CL004 is also satisfactorily reproduced by the fitted function, and the cluster parameters derived in this way are $r_{\mathrm{t}}=1.7$ and $c=0.75$. On the other hand, the routine failed to return physically reasonable parameters for VVV CL002, because the density between 0.5 and 1.5 is too high, causing $r_{\mathrm{t}}$ to diverge. A shallower slope of the density profile indicates the presence of tidal tails (Küpper et al. 2010b), and the cluster may be losing stars along its orbit. We therefore estimated $r_{\mathrm{t}}=1.8$ from the point where $N(r<d) / N_{\text {tot }}=1$ in Fig. 5, then a King profile of fixed tidal radius was fitted to find $c=0.65$. Similar problems were found with a Wilson (1975) profile, calculated from the energy distribution function through the code of Sollima et al. (2009): fitting both the central decay $(r \leq 0.5)$ and the enhanced tail was impossible. In this case the fitting routine converged, but to a very high tidal radius ( $c=0.68, r_{\mathrm{t}}=4.8$ ), and the resulting fit was unsatisfactory. We found that a EFF power-law (Elson et al. 1987) fits the observed profile well, with central density $\mu_{0}=5630$ stars, normalization radius $a=0$.' 16 , and power $-0.086(\gamma=0.17)$. The fitting routine likely underestimates the uncertainties associated with its output, because it does not take into account any source of systematic error, mainly the definition of the background level. We consider that the width of the bins of the density profile $(0.1)$ is a better estimate of the errors on the core, half-light, and tidal radii. Indeed, we found that these quantities can vary by up to 0 ' 1 from the given values when different but still reasonable assumptions on the background level were adopted.

\subsection{Color-magnitude diagrams}

The color-magnitude diagrams (CMDs) of the three objects are shown in the left panels of Fig. 6, where the cluster stars are overplotted on the photometry of a nearby comparison stellar field. The cluster area of VVV CL002 and VVV CL004 was limited to a circle of radius equal to the half-light radius to minimize the background contamination. The comparison field was an annulus of the same center and area as the cluster, and inner radius of 1 '.8, i.e. just outside the cluster tidal radius. A similar definition of cluster and field areas for VVV CL003 was complicated because its photometric catalog lacked the required symmetry about the center. We adopted as cluster region a circle of radius 0'.5, but part of this area fell outside the CCD. The definition of the comparison field was also complicated because VVV CL003 is found in a region affected by a very strong reddening gradient. Indeed, the interstellar extinction varies by up to $\Delta E(B-V) \approx 2$ over the $10^{\prime}$ of the chip, as confirmed by inspection of the Schlegel et al. (1998) maps. We noticed, however, that the CMD of the cluster area presents two well-defined red giant branches (RGBs): a narrow and well-populated one at $\left(J-K_{\mathrm{s}}\right) \approx 2$, and a broader RGB about 0.5 mag redder. The bluer sequence was found in all studied areas, always very similar in terms of quantity of stars, width, and magnitude of the red clump, although shifted to different colors. The other RGB component, on the other hand, was not observed outside $\sim 1^{\prime}$ from the cluster center. We therefore concluded that the bluer RGB represents the field population, and we searched for a region in the vicinity of the cluster where the color of this feature coincided with that observed in the cluster area. The adopted region had the same shape and dimension as the defined cluster area, 

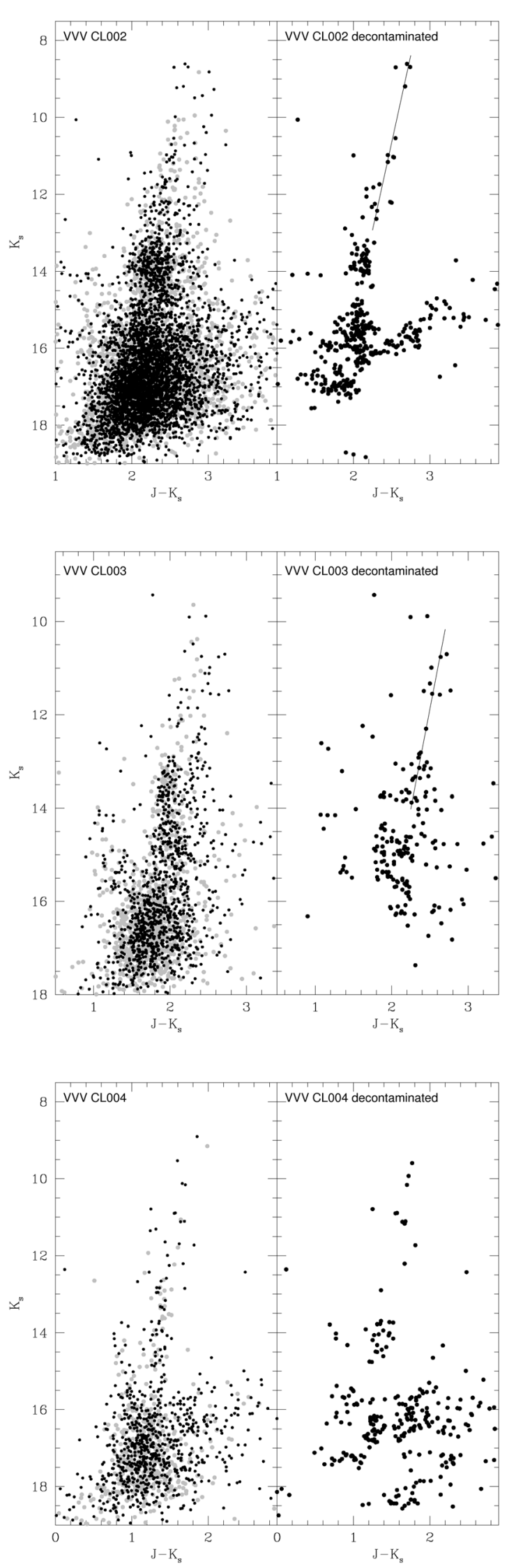

Fig. 6. Left panels: CMDs of the three cluster candidates (black dots) and their comparison fields (gray dots). Right panels: CMDs after statistical subtraction of the field contaminants.

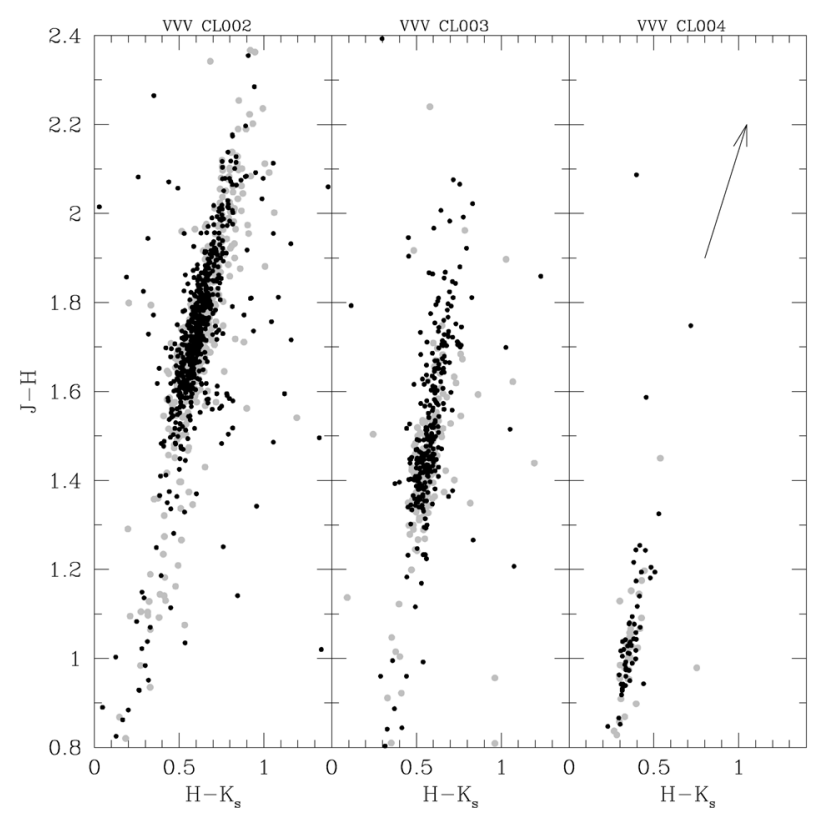

Fig. 7. Color-color diagrams of the three cluster candidates (black dots) and their comparison fields (gray dots). The plot was restricted to stars brighter than $K_{\mathrm{s}}=15$. The arrow in the right panel shows the direction of the reddening vector.

but offset $1^{\prime}$ toward the northwest, i.e. perpendicularly to the Galactic plane toward increasing Galactic latitude. Part of the object could still fall in this area, but its density should be very low at this distance. In Fig. 7 we show the color-color diagrams of the three objects and their comparison fields. This plot was restricted to stars brighter than $K_{\mathrm{s}}=15$ to highlight the color differences at the RGB level, because the increased photometric errors blur them for fainter stars.

As already noted, the CMD of VVV CL003 shows two distinct RGB sequences, and the redder one is not observed in the surrounding stellar field. In the comparison field, some residual cluster stars are also visible, not observed at longer distances from its center. On the other hand, the CMDs of VVV CL002 and VVV CL004 show no immediately clear difference with their comparison field, except for the enhanced number of stars. Further inspection reveals that the cluster RGB of VVV CL002 is on average bluer than the field population. This is visible even in the color-color diagrams, where cluster stars of VVV CL002 are offset to slightly bluer colors with respect to the field population. We found no color gradient that can explain this behavior in the whole $\sim 10^{\prime} \times 10^{\prime}$ area covered by our photometry, although the reddening pattern is probably variable on a small scale, as indicated by some highly reddened stars found in both cluster and comparison fields. VVV CL004 is less clear than the other two objects. Its color-color diagram contains more red stars than the surrounding field, but this is a simple consequence of the higher number of stars in the upper (redder) RGB of the cluster area. Indeed, although some bright $\left(K_{\mathrm{S}}=11-13\right)$ red stars without field counterparts are observed, the CMD of VVV CL004 shows no clear peculiarity with respect to the field, except for being much richer in brighter stars, as well as in faint red stars.

\subsection{Statistical field decontamination}

The CMDs of Fig. 6 are strongly contaminated by field stars, and so we applied a statistical procedure to isolate the cluster CMDs. 
The code written for this purpose was based on the method of Gallart et al. (2003). In brief, a metric was defined in the $K_{\mathrm{S}}-$ $\left(J-K_{\mathrm{s}}\right)$ plane as

$d=\sqrt{\left(K_{\mathrm{s}, \mathrm{a}}-K_{\mathrm{s}, \mathrm{b}}\right)^{2}+\left(k \times\left(\left(J-K_{\mathrm{s}}\right)_{a}-\left(J-K_{\mathrm{s}}\right)_{b}\right)\right)^{2}}$,

where $k$ is an arbitrary coefficient weighting a difference in color with respect to a difference in $K_{\mathrm{s}}$. The code scanned the list of stars in the comparison field and found the nearest star (smaller $d$ ) in the CMD of the cluster area for each object. This source was rejected as a contaminating field star if its distance $d$ was smaller than an arbitrary threshold $d_{\max }$, otherwise the star in the comparison area was flagged as an "object without a counterpart". In the first case, the list of stars in the cluster area was updated after the rejection before proceeding to the next entry in the list.

The choice of the parameters $d_{\max }$ and $k$ is not completely free of arbitrariness. Gallart et al. (2003) used $d_{\max }=0.3 \mathrm{mag}$, and we found this is a good choice in our case also, because it prevented the association between two stars with a very different position in the CMD. The same authors adopted $k=7$ in their optical photometry, which gives a dominant role to an offset in color. Their choice was dictated by the need to remove foreground contaminants distributed in a wide range of distances, with only limited variations of reddening. In our case, though, the patchy and locally variable structure of the reddening makes a color offset between cluster area and comparison field more likely than a difference in magnitude. Moreover, $\left(J-K_{\mathrm{s}}\right)$ is more sensitive than the $K_{\mathrm{s}}$ band to interstellar absorption, and even a different distance can affect the stellar color in our case, because we are observing through the dense clouds of the inner Galaxy. In conclusion, a much lower value of $k$ should be appropriate in our study. Indeed, we explored the effects of this choice through repeated trials, searching for the value that returned a clearer CMD and a smaller fraction of comparison field stars that were without counterpart. We found that both the residual contamination in the product CMDs and the quantity of unmatched field stars decreased with $k$, and $k \leq 2$ was clearly preferred. Only negligible changes were observed when the scaling factor was varied in the range $k=1-2$, and we finally adopted $k=1.6$. The unmatched field stars thus decreased to $\sim 2 \%$ for VVV CL004, which is below the Poissonian noise, while it was $\sim 10 \%$ for VVV CL002 and VVV CL003, i.e. about 3-4 times the Poissonian noise. However, the decontaminated CMDs of VVV CL002 and VVV CL003 contain 453 and 211 stars, respectively, which matches the expectation of 454 and 250 objects well, that was a result of the stellar counts of Sect. 3.3. Hence, the decontamination procedure worked satisfactorily.

In the decontamination procedure, different choices of the cluster and field areas necessarily led to different sets of removed stars. However, all features observed in the resulting CMDs, such as sequences and clumps, were observed in all cases when the cluster center was unchanged. The innermost regions of the three objects were excluded from the calculation, because our photometric catalogs could be incomplete in the most crowded regions. In general, the cleaning procedure left some residual field contamination, that decreased when choosing i) smaller areas, which consequently reduced the number of contaminating stars to be removed; and ii) a comparison field closer to the cluster center, which minimized the effects of a different local reddening. In both cases, the lower level of residual contamination came at the cost of a poorer derived CMD, because cluster members were also excluded. Nevertheless, these choices are justified here, because our aim is to unveil the behavior of the cluster CMD, while the true number of stars in the different CMD branches (i.e. the detailed luminosity function) is of lesser importance. Finally, the cluster area adopted in the decontamination procedure of VVV CL002 and VVV CL004 was a circle of radius 0.5 and 0.3 , respectively, slightly smaller than their halflight radii. The inner 0.'1 from the center was also excluded. The comparison fields were annuli of equal area as the cluster region, with inner radius of 0.9 in the case of VVV CL002 and 0.7 for VVV CL004. The cluster density is very low at these distances, and the loss of cluster stars in the resulting CMDs should be limited. Yet the areas of VVV CL003 and its comparison field were defined as in Sect. 3.4 (two circles of 0.5 radius, offset by $1^{\prime}$ ) because the strong underlying reddening gradient and the position of the cluster at the edge of the CCD gave no freedom for exploring different choices. Even for VVV CL003 the inner region with $r \leq 0$ ' 1 from the center was excluded.

The decontaminated CMDs of the three cluster candidates are shown in the right panels of Fig. 6. In all cases a clear evolutionary sequence of stars is visible, with a clumpy behavior caused by some underpopulated regions, and many highly scattered stars mainly at the fainter end of the diagrams. These features are probably an artifact of the decontamination procedure: when the cluster and field CMDs are very similar and the sample is statistically small, stars in crowded regions of the CMD are more easily matched with a field counterpart than the objects deviating because of high random error or higher differential reddening. As a consequence, the algorithm could oversubtract stars along the stellar sequence and under-subtract them in the wings of the color distribution, while still subtracting the expected amount of field contaminants, as indicated by the quantity of cluster and unmatched field stars given before.

\subsection{Reddening-free indices}

In the presence of non-uniform, spatially variable reddening, it is instructive to investigate the CMDs where the color and magnitude are substituted by reddening-free indices. In Fig. 8 we show the decontaminated CMDs of the three objects overplotted on their comparison field, with the indices $c_{3}=(J-H)-1.47 \times$ $\left(H-K_{\mathrm{s}}\right)$ and $m_{4}=K_{\mathrm{s}}-1.22 \times(J-H)$ in place of the color and magnitude. These quantities were defined by Catelan et al. (2011) assuming the Cardelli et al. (1989) relation between extinction in different bands, and $R=A_{\mathrm{V}} / E(B-V)=3.09$ (Rieke $\&$ Lebofsky 1985). We also experimented with analogous indices derived assuming the near-IR extintion law for the central Galactic regions (Nishiyama et al. 2009), but they led to the same general conclusions. Unfortunately, the error on $c_{3}$ and $m_{4}$ blurs the resulting diagram, because it is about three times larger than that in $\left(J-K_{\mathrm{s}}\right)$ and $K_{\mathrm{s}}$ respectively, owing to propagation of errors. The average uncertainty on these indices is about $0.1 \mathrm{mag}$ down to $m_{4}=10,0.15 \mathrm{mag}$ at $m_{4}=12$, and it increases exponentially at fainter magnitudes. Thus most of the information is erased. Moreover, while zeroing the effects of reddening, the indices also strongly limit the effect of other parameters, such as age or metallicity. For example, we verified through synthetic isochrone experiments that the RGBs of two stellar populations of the same age but different metallicity, with $[\mathrm{Fe} / \mathrm{H}]=0.0$ and -1.5 , would form two nearly parallel sequences separated by less than $\Delta c_{3} \approx 0.2$. This, combined with the large errors, makes two different populations difficult to distinguish. Nevertheless, Fig. 8 shows that the cluster sequence is redder than the field in VVV CL003. For VVV CL002 the difference is less evident, but both a linear and a quadratic fit of the upper RGB $\left(m_{4} \leq 12.5\right)$ are between 0.01 and 0.04 mag bluer in the cluster than in the 


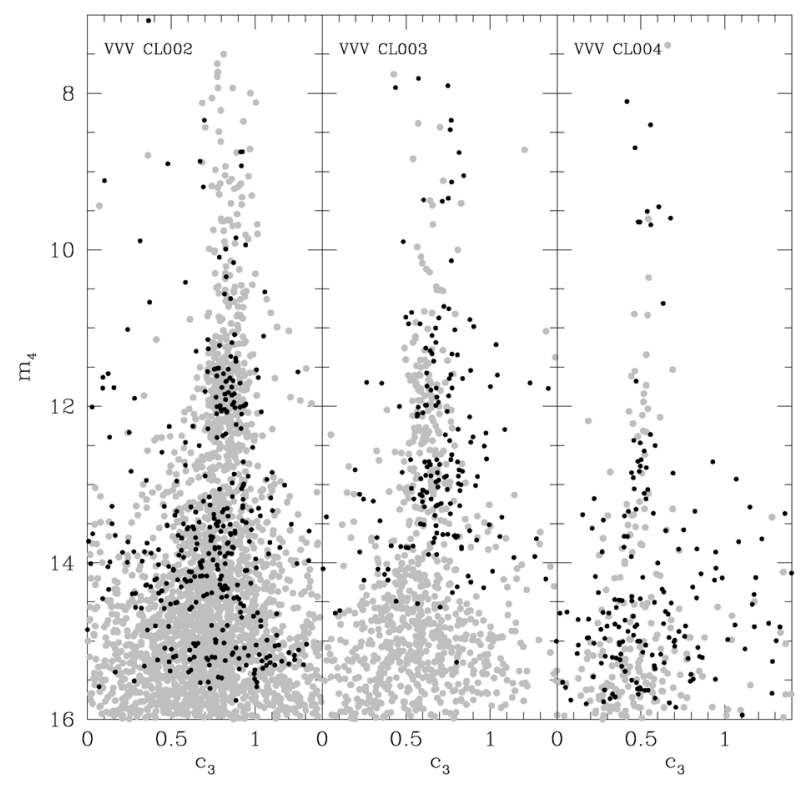

Fig. 8. Reddening-free CMDs of the three field decontaminated cluster candidates (black dots), overplotted on their comparison fields (gray dots).

field. Then, the color differences between these objects and their surrounding fields are not caused by differential reddening alone.

Figure 8 does not reveal much about VVV CL004, because field and cluster sequences already overlapped in the $\left(J-K_{\mathrm{s}}\right)-K_{\mathrm{s}}$ plane. It can be noted, however, that some red stars in the upper RGB ( $K_{\mathrm{s}}=11-13$, see Fig. 6) agree with the field locus in the $c_{3}-m_{4}$ diagram, indicating that some differential reddening is present even in the direction of this object.

\subsection{Proper motions}

The information about proper motion of stars in the field of an observed stellar overdensity is a very powerful tool to clarify its nature, especially if combined with the photometric data (e.g., Carraro 2005; Pavani \& Bica 2007). Indeed, a study of new cluster candidates without an analysis of the proper motions would seem incomplete. Unfortunately, it provides no real information for our three objects, because available data are scarce, and at the estimated distances ( $\sim 10 \mathrm{kpc}$, see Sects. 3.9-3.10) a too high precision is required to detected an appreciable difference with the field population. For example, the UCAC3 (Zacharias et al. 2010) and NOMAD (Zacharias et al. 2004) catalogs contain only 2 and 11 stars, respectively, in the inner 1' of VVV CL002.

The proper motions of stars in the direction of VVV CL003 and VVV CL004 and in their surrounding fields drawn from the PPMXL catalog (Roeser et al. 2010) are compared in the middle and lower panel of Fig. 9. The cluster stars were taken within 0'.8 (VVV CL003) and 0.5 (VVV CL004) from the center, and the comparison field was defined as an annulus between $2^{\prime}$ and $5^{\prime}$. Clearly, this comparison is inconclusive, and the average value in the cluster and field area do not differ by more than 1 mas $\mathrm{yr}^{-1}$ in both components. According to our stellar counts, only $11 \%$ and $13 \%$ of the stars in the selected region should be members of VVV CL003 and VVV CL004, respectively. Attempting to isolate cluster members of VVV CL003 through the 2MASS
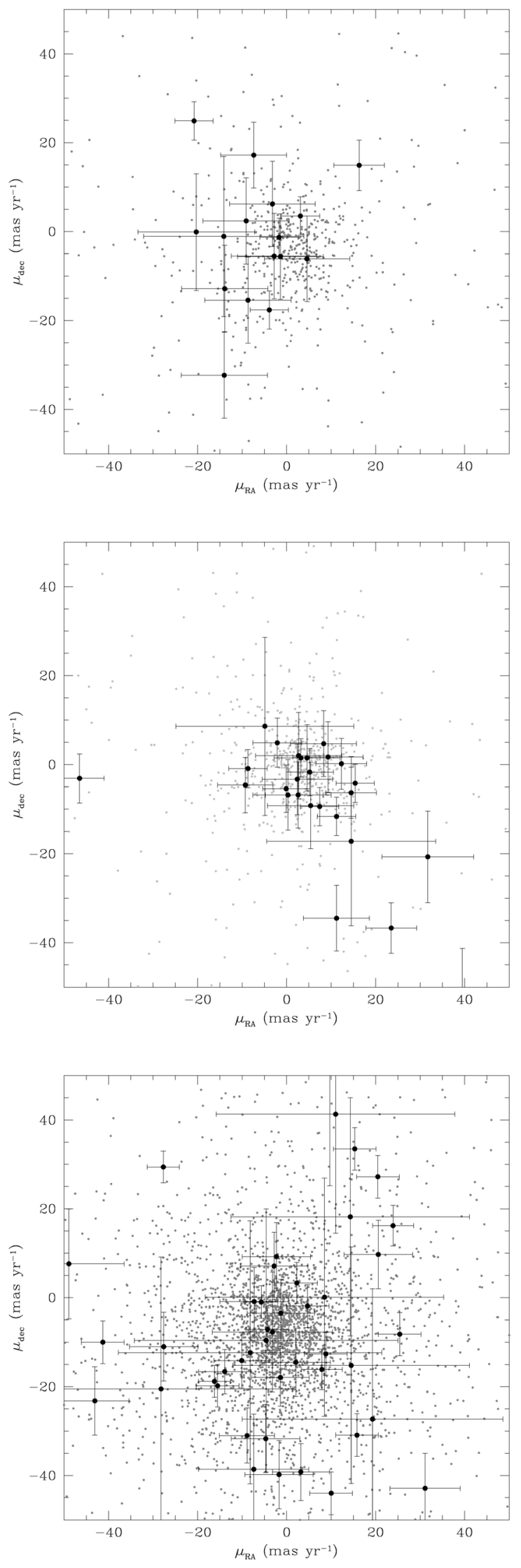

Fig. 9. Distribution of proper motions in the cluster area (full dots with error bars) and in the comparison field (small gray dots). Upper panel: VVV CL002; middle panel: VVV CL003; lower panel: VVV CL004. 
photometry given in the PPMXL catalog, only three stars compatible with the cluster sequence can be identified.

The proper motions of stars within 0.8 from the center of VVV CL002 are offset with respect to the comparison field, as shown in the upper panel of Fig. 9. Only 4.4 cluster members $(\sim 20 \%)$ are expected among the 22 entries of the PPMXL catalog, and its very hard to assess the significance of the observed behavior. As an exercise, we will check if this offset is compatible with the detection of the peculiar movement of VVV CL002. Assuming the escape velocity from the Galactic bulge ( $600 \mathrm{~km} \mathrm{~s}^{-1}$, Lamb et al. 1996) as an upper limit for the tangential velocity, the proper motion of stars at $7.5 \mathrm{kpc}$ from

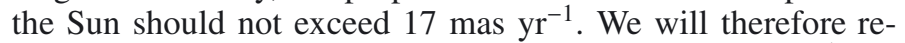

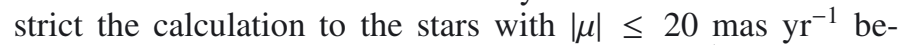

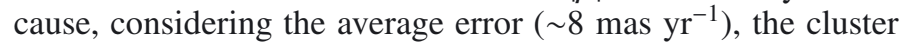
stars should not be found outside this range. The average values are $\left(\overline{\mu_{\alpha}}, \overline{\mu_{\delta}}\right)=(-2.8,-0.5)$ and $(0.8,-3.0)$ for the cluster and field stars, respectively. In the cluster area we can write $N_{\text {tot }} \bar{\mu}=$ $N_{\mathrm{f}} \overline{\mu_{\mathrm{f}}}+N_{\mathrm{c}} \overline{\mu_{\mathrm{c}}}$ for both components of proper motion, where the subscripts $f$ and $c$ denote the field and cluster members, respectively, and $N$ is the number of stars. We have $N_{\text {tot }}=12$, and assuming $N_{\mathrm{c}}=5, N_{\mathrm{f}}=7$, we obtain $\left(\overline{\mu_{\alpha}}, \overline{\mu_{\delta}}\right)=(-7.7,3.0)$ for cluster stars, which translates into a heliocentric tangential velocity of $\sim 300 \mathrm{~km} \mathrm{~s}^{-1}$ at $7.5 \mathrm{kpc}$. This is somewhat high, but an acceptable value. The observed behavior of proper motions in the field of VVV CL002 can therefore reflect the presence of a comoving group of stars.

\subsection{The nature of the cluster candidates}

Although the observed radial density profiles are well-represented by the King law, which is typical of stellar clusters, and the stellar overdensity associated with VVV CL002 and VVV CL003 is statistically highly significant, we will not rely on stellar counts alone to conclude about the nature of the three cluster candidates, because they can sometimes be misleading (see, for example, Villanova et al. 2004; Moni Bidin et al. 2010). Unfortunately, the proper motions do not provide useful information about this.

The case of VVV CL003 is straightforward, because the cluster sequence in the CMD is clearly distinct from the field one, hence this object cannot be only a fluctuation of the field stellar density. The redder putative cluster sequence cannot be ascribed to a local fluctuation of reddening, because in this case the extinction would be higher: a lack of stars would thus be expected instead of the observed significantly higher stellar counts. The reddening-free CMD also supports this conclusion.

The significance of the stellar overdensity detected in the direction of VVV CL002 is the highest among the three candidates. As an additional proof that the cluster and field stars originate from different populations, we derived the spatial density distribution of the stars with $K_{\mathrm{s}} \leq 17$ located within $\left|\Delta\left(J-K_{\mathrm{s}}\right)\right| \leq$ 0.07 from the isochrone plotted in Fig. 12, which fits the decontaminated cluster sequence with the parameters derived in Sect. 3.9. The resulting density map is shown in the upper panel of Fig. 10, where each $2^{\prime \prime} \times 2^{\prime \prime}$ pixel is assigned the average density in the $20^{\prime \prime} \times 20^{\prime \prime}$ square centered on it. The local overedensity associated to the cluster is clearly visible at $(x, y) \approx(250,750)$. The procedure was then repeated, shifting the same isochrone by 0.14 mag toward the red. The map is shown in the lower panel of the same figure, where the cluster overdensity is barely visible, lost in the patchy structures of the field. A random overdensity of stars should be observed in any region of the CMD where the field is found though. This demonstrates that VVV CL002 is
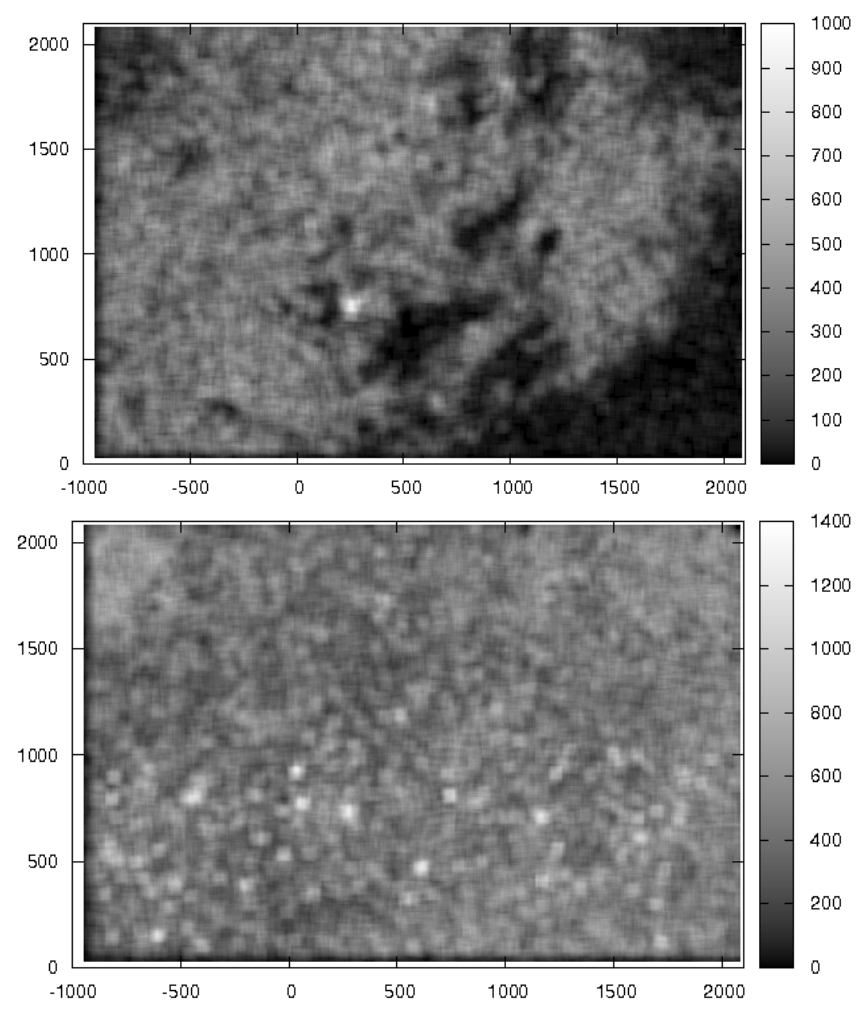

Fig. 10. Upper panel: spatial density of stars with $K_{\mathrm{s}} \leq 17$ located within $\left|\Delta\left(J-K_{\mathrm{s}}\right)\right| \leq 0.07$ from the isochrone of Fig. 12 for VVV CL002. The coordinates are given in pixels, and the density is in units of stars per $\operatorname{arcmin}^{2}$. Lower panel: same as the upper panel, but with an isochrone shifted 0.14 mag toward the red.

not a simple fluctuation of the field density. Still, we could be in the presence of a narrow, deep window in the interstellar clouds, where a higher quantity of stars, on average bluer because less reddened, would be found. However, the presence of a similar color offset even in the reddening-free CMD argues against this hypothesis. Therefore, VVV CL002 and VVV CL003 are most likely two new stellar clusters.

Even VVV CL004 is a simple case, because all evidence points at a random fluctuation of field stars. Indeed, local stellar clustering is not very significant, its stars are photometrically indistinguishable from the field population, and its radial density profile, although well fitted with a King profile, is quite peculiar. Nevertheless, VVV CL004 could still be an open cluster remnant (OCR), a dynamically evolved cluster in the last stage before dissolution. Open cluster remnants should appear as small groups of bright stars, depleted of the fainter main-sequence objects (de La Fuente Marcos 1997, 1998), as can be observed in the field-decontaminated CMD of VVV CL004.

In the following section we will estimate the physical parameters of VVV CL002 and VVV CL003. The results are summarized in Table 3. A similar analysis is not presented for VVV CL004, both because the object is most probably not a real cluster, and because the stars in the decontaminated CMD are too few and dispersed for a reliable estimate. The most satisfactory isochrone fit of the VVV CL004 CMD is shown in Fig. 12. It was obtained assuming $[\mathrm{Fe} / \mathrm{H}]=-1.1,[\alpha / \mathrm{Fe}]=+0.30$, $(m-M)_{K_{\mathrm{s}}}=15.4, E\left(J-K_{\mathrm{s}}\right)=0.82$, and an age of $7.5 \mathrm{Gyr}$. It is not the only solution, anyway, and other similar fits can be obtained with a very different set of parameters.

The transformations of Grocholski \& Sarajedini (2002) were used to link the theoretical $K$ values to the observed 


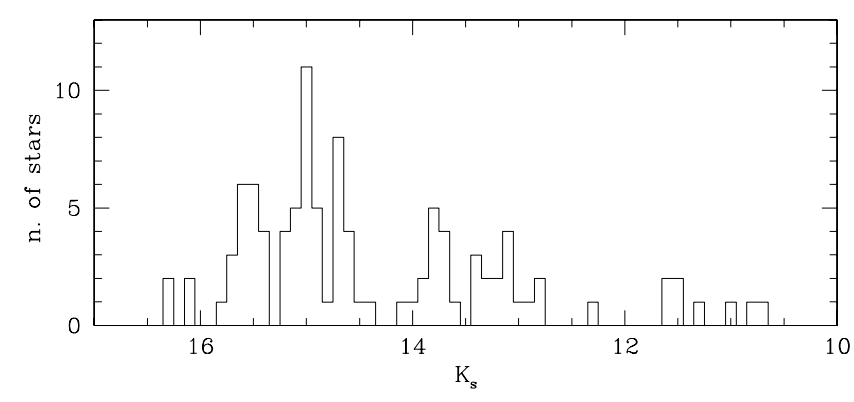

Fig. 11. Luminosity function of RGB stars in VVV CL003.

Table 3. Derived parameters of the two cluster candidates.

\begin{tabular}{lcc}
\hline \hline & VVV CL002 & VVV CL003 \\
\hline$r_{\mathrm{h}}\left(^{\prime}\right)$ & $0.75 \pm 0.10$ & $0.6 \pm 0.1$ \\
$r_{\mathrm{h}}(\mathrm{pc})$ & $1.6 \pm 0.3$ & $2.3 \pm 0.4$ \\
$r_{\mathrm{t}}\left({ }^{\prime}\right)$ & $1.8 \pm 0.1$ & $1.8 \pm 0.1$ \\
$r_{\mathrm{t}}(\mathrm{pc})$ & $3.8 \pm 0.5$ & $6.8 \pm 0.4$ \\
$c=\log \left(r_{\mathrm{h}} / r_{\mathrm{c}}\right)$ & $0.65 \pm 0.26$ & $0.56 \pm 0.21$ \\
$(m-M)$ & $14.32 \pm 0.23$ & $15.57 \pm 0.16$ \\
$d(\mathrm{kpc})$ & $7.3 \pm 0.9$ & $13.0 \pm 1.0$ \\
$R_{\mathrm{GC}}(\mathrm{kpc})$ & $0.7 \pm 0.9$ & $5.0 \pm 1.0$ \\
$z(\mathrm{pc})$ & $113 \pm 13$ & $166 \pm 14$ \\
$E\left(J-K_{\mathrm{s}}\right)$ & $1.50 \pm 0.15$ & $1.48 \pm 0.15$ \\
$E(B-V)$ & $2.88 \pm 0.29$ & $2.85 \pm 0.29$ \\
{$[\mathrm{M} / \mathrm{H}]$} & $-0.16 \pm 0.2$ & $-0.1 \pm 0.2$ \\
{$[\mathrm{Fe} / \mathrm{H}]$} & $-0.4 \pm 0.2$ & $-0.1 \pm 0.2$ \\
$M_{V}$ & $-3.4 \pm 0.3$ & - \\
Age $(\mathrm{Gyr})$ & $\geq 6.5$ & - \\
\hline
\end{tabular}

$K_{\mathrm{s}}$ magnitudes. We relied on key photometric quantities measured on the decontaminated CMDs, through the calibrations of Valenti et al. (2004b) and Ferraro et al. (2006, hereafter F06). In particular, F06 define two sets of equations for disk/halo-like and bulge-like objects, depending on the assumed trend of the $\alpha$ enhancement with metallicity. Indeed, the $\alpha$-element abundance affects the location and curvature of the RGB, which are better described as a function of the so-called global metallicity $([\mathrm{M} / \mathrm{H}])$ which, for $[\mathrm{Fe} / \mathrm{H}] \leq-1$, can be expressed as

$[\mathrm{M} / \mathrm{H}]=[\mathrm{Fe} / \mathrm{H}]+\log \left(0.638 f_{\alpha}+0.362\right)$,

where $f_{\alpha}$ is the $\alpha$-elements enhancement factor (Salaris et al. 1993). This relation, however, breaks down with increasing metallicity (VandenBerg et al. 2000; Kim et al. 2002).

\subsection{VVV CL002 parameters}

The decontaminated CMD of VVV CL002 shows a clump of stars at $K_{\mathrm{s}} \approx 14$. We interpret this as a signature of the cluster's horizontal branch red clump. Following the prescriptions of Kuchinski \& Frogel (1995) and Kuchinski et al. (1995), we linearly fitted the upper RGB from 0.6 mag above the clump up to the brightest stars, obtaining a slope of -0.110 . The fit is shown in Fig. 6. This value is very similar to the slope of the RGB of NGC 6528 (Ferraro et al. 2000) and Liller 1 (Valenti et al. 2010), two metal-rich bulge globular clusters enriched in $\alpha$-elements $([\alpha / \mathrm{Fe}] \approx+0.30)$. NGC 6528 is the bulge $\mathrm{GC}$ richest in iron, with $[\mathrm{Fe} / \mathrm{H}]$ estimates in the range $-0.1-+0.1$ (Zoccali et al. 2004; Origlia et al. 2005; Carretta et al. 2009), while Origlia et al. (2001) report $[\mathrm{Fe} / \mathrm{H}]=-0.30$ for Liller 1 . For VVV CL002 we used the F06 equations for bulge systems, because of its location very close to the Galactic center (see later), obtaining $[\mathrm{M} / \mathrm{H}]=-0.16 \pm 0.20$ and, assuming $[\alpha / \mathrm{Fe}]=+0.30$, $[\mathrm{Fe} / \mathrm{H}]=-0.4 \pm 0.2$ from Eq. (4). The errors were derived from the uncertainties in the equation constants estimated by Ferraro et al. (2000), which dominate the error budget when compared to the uncertainties in the derived slope. Had we used the equations for disk/halo objects, we would have obtained $[\mathrm{M} / \mathrm{H}]=-0.24$ and $[\mathrm{Fe} / \mathrm{H}]=-0.45$, consistent with the adopted values.

The mean magnitude and color of the stars in the red clump of VVV CL002 are $K_{\mathrm{s}}=13.80 \pm 0.15$ and $\left(J-K_{\mathrm{s}}\right)=2.17 \pm 0.04$, where the rms of the measurements are taken as uncertainties on the average values. The absolute magnitude of the red clump weakly depends on the age and metallicity of the stellar population. Ferraro et al. (2006) propose $M_{K, 0}=-1.40$ and a conservative error to take this effect into account, but still their calibration is more suited for intermediate metal-poor, old clusters. VVV CL002 is at the upper limit of the metallicity range considered by F06, and its red clump is therefore expected to be brighter, as indicated by the theoretical values tabulated by Salaris \& Girardi (2002). Indeed, Alves (2000) report a mean value 0.2 mag brighter than F06 for their solar-metallicity field sample. We finally adopted $M_{K, 0}=-1.50 \pm 0.15$ which, as shown by Table 1 of Salaris \& Girardi (2002), encompasses the theoretical expectations for stars at $[\mathrm{Fe} / \mathrm{H}]=-0.4$ in the age interval from 1 to $11 \mathrm{Gyr}$. We thus obtain the apparent distance modulus $(m-M)_{K_{\mathrm{s}}}=15.34 \pm 0.21$. From Eq. (3.4) of F06, it follows that the apparent magnitude at the unreddened color $\left(J-K_{\mathrm{s}}\right)_{0}=0.7$ is $K_{\mathrm{s}}=13.52$. The observed color at this magnitude was obtained with a linear fit of the cluster RGB, restricted to the range $K_{\mathrm{s}}=13-14$ to limit the effects of the RGB curvature. The comparison of this value with the expectation for null reddening gave $E\left(J-K_{\mathrm{s}}\right)=1.50 \pm 0.15$. The uncertainty associated to the fit returned a too small error $( \pm 0.03 \mathrm{mag})$, while the reddening of Galactic GCs is usually uncertain to at least 10\% (see, for example, the discussion in Harris 1996, 2010 version $)$. The error on $E\left(J-K_{\mathrm{s}}\right)$ was therefore arbitrarily fixed to the more realistic value given above. The reddening agrees well with the value obtained by horizontally shifting a PGPUC isochrone (Valcarce 2011; Valcarce et al. in prep.), with the required metallicity, $\alpha$-enhancement, and apparent distance modulus, to find the best fit of the observed RGB. Through the transformations of Cardelli et al. (1989), the derived reddening translates into $E(B-V)=2.88 \pm 0.29, A_{K_{\mathrm{s}}}=1.02 \pm 0.10$, implying a true distance modulus $(m-M)_{0}=14.32 \pm 0.23$ and a distance $d=7.3 \pm 0.9 \mathrm{kpc}$. Had we used the mean intrinsic color of the red clump proposed by López-Corredoira et al. (2002), we would have obtained $E\left(J-K_{\mathrm{s}}\right)=1.46 \pm 0.15$, in good agreement with our estimate, but in this case the final isochrone fit of the cluster CMD is of lesser quality. Schlegel et al. (1998)'s maps give $E(B-V)=3.47$ in the direction of VVV CL002, but this higher value should not be surprising, because it refers to the total Galactic absorption, while the cluster should be located only half way to the end of the absorbing gas column. In any case, the corrections proposed by Bonifacio et al. (2000) give $E(B-V)=2.29$, a value incompatible with our results.

At a distance of $7.3 \mathrm{kpc}$, the tidal and half-light angular radii of VVV CL002 (Sect. 3.3) translate into the physical dimensions $3.8 \pm 0.5$ and $1.6 \pm 0.3 \mathrm{pc}$, respectively. From the estimated distance and the Galactic coordinates $(l, b)$, assuming a solar Galactocentric distance $R_{\odot}=8 \mathrm{kpc}$, we also derived the distance from the Galactic center $R_{\mathrm{GC}}=0.6 \pm 0.8 \mathrm{kpc}$, and the height above the Galactic plane $z=113 \pm 12 \mathrm{pc}$.

The cluster age was determined by fitting the lower part of the decontaminated CMD with a PGPUC isochrone with the metallicity, $\alpha$-enhancement, distance modulus, and reddening 


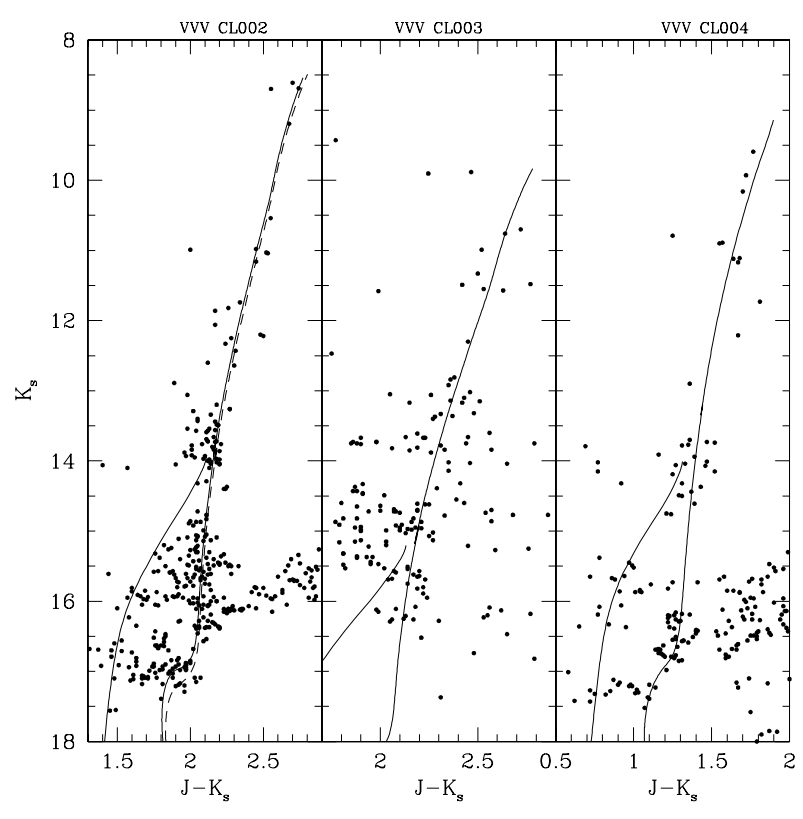

Fig. 12. Decontaminated CMDs of the three clusters, with the bestfitting PGPUC isochrones defined in the text overplotted. The zero-age horizontal branch (ZAHB) in all panels of the figure was calculated with the helium abundance $Y=0.245$. For VVV CL002, two isochrones at 6.5 Gyr (thick curve) and 8.5 Gyr (dashed curve) are shown.

determined above. In Fig. 12 we show this fit with two isochrones of different age. We found that a young age $(\sim 6.5 \mathrm{Gyr})$ was required to fit the feature at $\left(K_{\mathrm{s}},\left(J-K_{\mathrm{s}}\right)\right) \approx$ $(17,2)$. This clump of stars, closely resembling the cluster's subgiant branch (SGB) and upper turn-off, was always observed when varying the cluster area and the comparison field in the decontamination procedure. However, we regard this age estimate as uncertain, because it completely relies on stars at the fainter end of our diagram, but it can be regarded as a lower limit: had the cluster been younger, the SGB would have been brighter, and thus more clearly observed in our photometry. Deeper data unveiling the turnoff region are required to confirm these results. It must also be remembered that we used isochrones with $Y=0.245$, but a different helium abundance can affect the age estimate, because stars with higher helium content evolve faster.

The cluster distance and all other distance-dependent parameters such as $R_{\mathrm{GC}}, z$, and the physical size, depend on the assumed absolute magnitude of the red clump. As an independent test of our results, we estimated the distance modulus differentially with respect to 47 Tucanae $([\mathrm{Fe} / \mathrm{H}]=-0.7$, Gratton et al. $2003)$, and NGC $6528([\mathrm{Fe} / \mathrm{H}] \approx 0)$, assuming the photometry by Valenti et al. (2004a) to derive the shift in color and magnitude between them and VVV CL002, and the Cardelli et al. (1989) extinction coefficients. Despite the different metallicity of the two comparison clusters, the resulting distance modulus differs by only one tenth of magnitude in the two cases. We obtain $(m-M)_{0}=14.16$, and a distance $d=6.8 \mathrm{kpc}$. that agrees within errors with our estimate. The difference is caused by the old age of the two comparison clusters (10-11 Gyr, Gratton et al. 2003; Ortolani et al. 2001): the distance modulus derived with this empirical method is 0.16 mag lower than what we previously obtained, and Salaris \& Girardi (2002) predict that the red clump of a population of similar age and $[\mathrm{Fe} / \mathrm{H}]=-0.4$ would be $0.12-0.15 \mathrm{mag}$ fainter than our assumption. We thus conclude that, although the two estimates agree well, a distance shorter by about $0.5 \mathrm{kpc}$ should be preferred if VVV CL002 is an old GC.
We estimated the total luminosity of the cluster integrating the flux collected within increasing apertures up to the tidal radius, and subtracting the expected field contribution. The measurements were performed with SExtractor (Bertin \& Arnouts 1996) on the stacked tile in the $J$-band, because many saturated stars prevented a reliable estimate in the $K_{\mathrm{s}}$-band frame. We thus found a total magnitude $J_{\text {tot }}=12.5 \pm 0.2$, and from the mean cluster color $\left(J-K_{2}\right)=2.3 \pm 0.1$ and the apparent distance modulus, we obtain $M_{K_{\mathrm{s}}}=-5.1 \pm 0.3$, which translates into $M_{V}=-3.4 \pm 0.3$ assuming the synthetic colors of an old stellar population calculated by Leitherer et al. (1999).

\subsection{VVV CL003 parameters}

The interpretation of the decontaminated CMD of VVV CL003 must take into account the problems that its photometry presented. The diagram reveals a clear sequence of stars distinct from the field population, resembling a cluster RGB. A sparse group of highly reddened stars is also visible to the right of this sequence. Many of them overlap with the cluster RGB in the reddening-free CMD and are probably the result of differential reddening, while some are most probably foreground cool dwarfs, because they still keep their redder color even in Fig. 8. On the bluer side, a residual contamination is found. There is a lack of stars fainter than $K_{\mathrm{s}}=16$, probably because almost the entire cluster area falls in the CCD border, where the data were collected in only one of the two jittered exposures forming a frame. The cluster RGB and the redder objects are visible for any definition of cluster and field area, provided that the latter is not more distant than $2-3^{\prime}$ from the center, while the residual contamination may change in color and quantity of stars depending on the location of the comparison field.

A dense group of stars in the cluster RGB is found at $K_{\mathrm{s}}=$ 15 . This feature is more easily observed plotting the histogram of the distribution of stars along the sequence, as shown in Fig. 11. In this plot we considered only stars within 0.2 mag in color from the RGB ridge line, defined as the isochrone plotted in Fig. 12. Unfortunately, the field and cluster RGBs are close in this part of the CMD, and the behavior of this feature (and even its detection) strongly depends on the choice of the comparison field. However, an enhanced residual contamination appeared at the same magnitude and a bluer color when the clump in the RGB was not found, indicating that the field RGB was too red, partially overlapping and erasing the cluster clump. We will therefore assume this group of stars is the cluster horizontal branch red clump, but we caution that the subsequent analysis is based on this detection, which is not clear beyond any doubt.

Repeating the same procedures used for VVV CL002 (Sect. 3.9), the slope of the upper RGB estimated with the fit shown in the right panel of Fig. 6 is -0.117 . This value is lower than the slope of any of the 27 Galactic GCs studied by Ferraro et al. (2000) and Valenti et al. (2004b, 2010), indicating a high metal content. The location of VVV CL003 in the Galactic disk outside the bulge (see below) led us to adopt the F06 calibration for disk objects and no $\alpha$-enhancement, obtaining $[\mathrm{M} / \mathrm{H}]=[\mathrm{Fe} / \mathrm{H}]=-0.1 \pm 0.2$. For the absolute magnitude of the red clump we opted for the value proposed by Alves (2000), given the high metallicity derived from the RGB slope. We thus obtained an apparent distance modulus $(m-M)_{K_{\mathrm{s}}}=16.58 \pm 0.12, E\left(J-K_{\mathrm{s}}\right)=1.48 \pm 0.15$ from Eq. (2.4) of F06, and $E(B-V)=2.85 \pm 0.29$. Using the calibration of López-Corredoira et al. (2002), we would have obtained $E\left(J-K_{\mathrm{s}}\right)=1.46$. On the contrary, Schlegel et al. (1998) give $E(B-V)=3.34$ in the direction of VVV CL003. As in the 

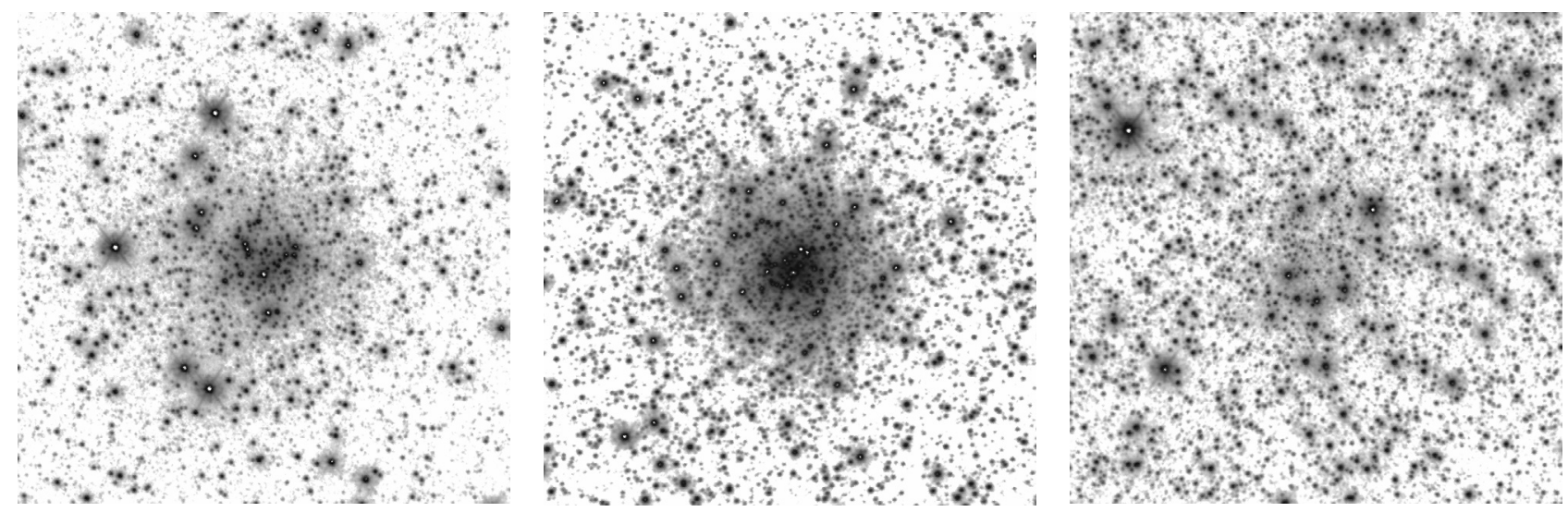

Fig. 13. 2! $4 \times 2$ '. logarithmic gray-scale $K_{\mathrm{s}}$-band images of (from left to right) NGC 6528, Liller 1, and VVV CL002, from the VVV archive. North is up and east is to the right.

case of VVV CL002, this value is too high compared with our estimates, while the corrections of Bonifacio et al. (2000) reduce it too much, returning $E(B-V)=2.19$. However, the resolution of the Schlegel et al. (1998) maps is too low for this region of the sky of strong reddening gradients, and it is not surprising that their average value in the resolution element does not coincide with the local absorption. It could also be that the relation $R=A_{\mathrm{V}} / E(B-V)=3.1$, required to transform the reddening in different bands through the relations of Cardelli et al. (1989), is not valid in this highly obscured part of the sky.

The reddening in the infrared bands implies $A_{K_{\mathrm{s}}}=1.01 \pm$ 0.10 , a true distance modulus $(m-M)_{0}=15.57 \pm 0.16$, and a distance $d=13 \pm 1.0 \mathrm{kpc}$. With simple geometrical arguments based on the $(l, b)$ coordinates given in Table 1 , we obtain the Galactic height $z=166 \pm 14 \mathrm{pc}$, and the Galactocentric distance $R_{\mathrm{GC}}=5.0 \pm 1.0 \mathrm{kpc}$. At a heliocentric distance of $13 \mathrm{kpc}$, the angular size of VVV CL003 translates into the physical quantities $r_{\mathrm{t}}=6.8 \pm 0.4 \mathrm{pc}$ and $r_{\mathrm{h}}=2.3 \pm 0.4 \mathrm{pc}$.

An age estimate for VVV CL003 is impossible, because the CMD does not reach the SGB. We found that the observed RGB could be satisfactorily fitted with isochrones of virtually any age, although lower values required a progressively higher reddening to match the observed sequence. For example, an isochrone of 2 Gyr better fitted the cluster CMD if $E\left(J-K_{\mathrm{s}}\right)=1.56$ is assumed. This is not necessarily a reason to prefer an older age for VVV CL003, because it could simply reflect the fact that the F06 relation used to estimate the reddening was calibrated on old stellar populations.

The high interstellar reddening gradient and the problematic photometric data (Sect. 2.2) prevented a reliable estimate of the cluster total luminosity.

\section{Discussion and conclusions}

The age of VVV CL002 is uncertain, but the proposed lower limit of $6.5 \mathrm{Gyr}$, its subsolar metallicity, and its height above the Galactic plane, twice the scale height of young objects (Joshi 2005), argue in favor of an old cluster. On the other hand, Friel (1995) showed that old open clusters are completely absent in the inner $\sim 7 \mathrm{kpc}$ from the Galactic center. Because of its location very close to the Galactic center, VVV CL002 is therefore most likely a GC.

So far, HP 1 is the closest known GC to the Galactic center $\left(R_{\mathrm{GC}}=0.5 \mathrm{kpc}\right.$ Barbuy et al. 2006), and only six GCs have been discovered in the inner $1 \mathrm{kpc}$ (Harris 1996). VVV CL002, with an estimated Galactocentric distance of $0.7 \mathrm{kpc}$, is therefore one of the innermost Galactic GCs. Curiously, the density profile of
VVV CL002 suggests the presence of tidal tails, a behavior more easily observed when the cluster is close to the perigalacticon (Küpper et al. 2010a). If the connection between tidal tails and perigalacticon were to be confirmed even for objects subject to the intense stress of the inner Galaxy, then the cluster orbit would be confined to the very central region of the Galaxy.

VVV CL002 has a global metallicity close to NGC 6528 and Liller 1, as indicated by the slope of the upper RGB. These two objects are also bulge GCs very close to the Galactic center $\left(R_{\mathrm{GC}}=0.6\right.$ and $0.8 \mathrm{kpc}$, respectively; Valenti et al. 2010; Feltzing \& Johnson 2002) and, as in the case of VVV CL002, their stars also are barely distinguished from the background bulge population in the CMD (Davidge 2000). However, the similarity cannot be drawn further, because their appearance is very distinct. In Fig. 13 we show a visual comparison between the three objects, with $K_{\mathrm{s}}$-band images from the VSA archive: VVV CL002 is clearly sparser and less massive, resembling a Palomar-like object more than a highly concentrated bulge GC such as NGC 6528 and Liller 1. Indeed, our estimate of its total luminosity is $\sim 3.5$ mag fainter than these two objects (Webbink 1985; Peterson \& Reed 1987; van den Bergh et al. 1991; Mallen-Ornelas \& Djorgovski 1993). VVV CL002 is more affine to moderately metal-rich, low-mass, and sparse GCs such as Whiting 1 and Palomar 1, whose physical size are comparable $\left(r_{\mathrm{h}}=1.9\right.$ and 1.5-2.2 pc, respectively; Harris 1996; Rosenberg et al. 1998). These two objects are relatively young (Rosenberg et al. 1998; Carraro 2005), and are only slightly more metalpoor (Carraro et al. 2007b; Monaco et al. 2011), although they are one magnitude brighter (Harris 1996). Palomar 12 also has a similar young age (Stetson et al. 1989; Geisler et al. 2007) but has a lower metallicity (Cohen 2004), and it is physically larger (Harris 1996). It is worth noting that both Whiting 1 and Palomar 12 are associated with the Sagittarius dwarf spheroidal galaxy (Irwin 1999; Carraro et al. 2007b; Law \& Majewski 2010), while the extragalactic origin of Palomar 1 has been repeatedly proposed although never proven (e.g., Crane et al. 2003; Frinchaboy et al. 2004; Belokurov et al. 2007).

VVV CL003 is surely a physical cluster of stars, located in the Galactic disk beyond the center, outside the bulge, at a Galactocentric distance of $5 \mathrm{kpc}$. Its high metallicity and its position in the Galaxy are more typical of an open cluster, although a GC cannot be excluded. Deeper photometry, reaching at least the cluster SGB two magnitudes fainter than the limit reached with our data, is needed to estimate its age and, thus, better clarify its nature. Interestingly enough, to our knowledge it is the first stellar cluster ever discovered on the "dark side of the Galaxy", 
i.e. the Galactic disk on the other side of the center. The Open Cluster Database 4 (WEBDA, Mermilliod 1996) has no entry in the direction of the bulge more distant than a few kpc.

VVV CL004 is most probably only an asterism, and as such it deserves no further attention. Nevertheless, the possibility of an OCR remains open, and this kind of object is of great interest, because we are still lacking observational evidence of the last stages of the cluster dynamical evolution. Indeed, OCRs are very elusive and easily confused with random field fluctuations and, despite a large list of candidates (e.g., Pavani \& Bica 2007; Bica et al. 2001), only one genuine OCR has been to date spectroscopically confirmed (NGC 1901, Carraro et al. 2007a).

Acknowledgements. We gratefully acknowledge use of data from the ESO Public Survey programme ID 179.B-2002 taken with the VISTA telescope, and data products from the Cambridge Astronomical Survey Unit. We acknowledge support by the FONDAP Center for Astrophysics 15010003, BASAL Center for Astrophysics and Associated Technologies PFB-06/2007, the Chilean Ministry for the Economy, Development, and Tourism's Programa Iniciativa Científica Milenio through grant P07-021-F, awarded to The Milky Way Millennium Nucleus, FONDECYT 1090213, 1080086, 1110393 and 1110326, and from CONICYT. This investigation made use of data from the Two Micron All Sky Survey, which is a joint project of the University of Massachusetts and the Infrared Processing and Analysis Center/California Institute of Technology, funded by the National Aeronautics and Space Administration and the National Science Foundation. We thank the referee, W. E. Harris, for improving the manuscript quality with his detailed comments.

\section{References}

Abazajian, K. N., Adelman-McCarthy, J. K., Agüeros, M. A., et al. 2009, ApJS, 182,543

Alves, D. R. 2000, ApJ, 539, 732

Barbuy, B., Zoccali, M., Ortolani, S., et al. 2006, A\&A, 449, 349

Belokurov, V., Evans, N. W., Irwin, M. J., et al. 2007, ApJ, 658, 337

Benjamin, R. A., Churchwell, E., Babler, B. L., et al. 2003, PASP, 115, 953

Bertin, E., \& Arnouts, S. 1996, A\&AS, 117, 393

Bica, E., Santiago, B. X., Dutra, C. M., et al. 2001, A\&A, 366, 827

Bica, E., Bonatto, C., Ortolani, S., \& Barbuy, B. 2007, A\&A, 472, 483

Bonatto, C., Bica, E., Ortolani, S., \& Barbuy, B. 2007, MNRAS, 381, L45

Bonifacio, P., Monai, S., \& Beers, T. C. 2000, AJ, 120, 2065

Borissova, J., Bonatto, C., Kurtev, R., et al. 2011, A\&A, 532, A131

Cardelli, J. A., Clayton, G. C., \& Mathis, J. S. 1989, ApJ, 345, 245

Carraro, G. 2005, ApJ, 621, L61

Carraro, G., de La Fuente Marcos, R., Villanova, S., et al. 2007a, A\&A, 466, 931

Carraro, G., Zinn, R., \& Moni Bidin, C. 2007b, A\&A, 466, 181

Carretta, E., Bragaglia, A., Gratton, R., D’Orazi, V., \& Lucatello, S. 2009, A\&A, 508,695

Catelan, M., Minniti, D., Lucas, P. W., et al. 2011, to appear in RR Lyrae Stars, Metal-Poor Stars, and the Galaxy, ed. A. McWilliam, Carnegie Obs. Astrophys. Ser., 5 [arXiv: 1105.1119]

Cohen, J. G. 2004, AJ, 127, 1545

Crane, J. D., Majewski, S. R., Rocha-Pinto, H. J., et al. 2003, ApJ, 594, L119

Davidge, T. J. 2000, ApJS, 126, 105

de La Fuente Marcos, R. 1997, A\&A, 322, 764

de La Fuente Marcos, R. 1998, A\&A, 333, L27

Elson, R. A. W., Fall, S. M., \& Freeman, K. C. 1987, ApJ, 323, 54

Emerson, J., \& Sutherland, W. 2010, The Messenger, 139, 2

Feltzing, S., \& Johnson, R. A. 2002, A\&A, 385, 67

Ferraro, F. R., Montegriffo, P., Origlia, L., \& Fusi Pecci, F. 2000, AJ, 119, 1282

Ferraro, F. R., Valenti, E., \& Origlia, L. 2006, ApJ, 649, 243

Friel, E. D. 1995, ARA\&A, 33, 381

Frinchaboy, P. M., Majewski, S. R., Crane, J. D., et al. 2004, ApJ, 602, L21

Froebrich, D., Meusinger, H., \& Davis, C. J. 2008a, MNRAS, 383, L45

Froebrich, D., Meusinger, H., \& Scholz, A. 2008b, MNRAS, 390, 1598

Gallart, C., Zoccali, M., Bertelli, G., et al. 2003, AJ, 125, 742

Geisler, D., Wallerstein, G., Smith, V. V., \& Casetti-Dinescu, D. I. 2007, PASP, 119,939

Gnedin, O. Y., \& Ostriker, J. P. 1997, ApJ, 474, 223

Gratton, R. G., Bragaglia, A., Carretta, E., et al. 2003, A\&A, 408, 529

Grocholski, A. J., \& Sarajedini, A. 2002, AJ, 123, 1603

Harris, W. E. 1996, AJ, 112, 1487
Irwin, M. 1999, in The Stellar Content of Local Group Galaxies, ed. P. Whitelock, \& R. Cannon, IAU Symp., 192, 409

Irwin, M. J., Lewis, J., Hodgkin, S., et al. 2004, in SPIE Conf. Ser. 5493, ed. P. J. Quinn, \& A. Bridger, 411

Ivanov, V. D., Kurtev, R., \& Borissova, J. 2005, A\&A, 442, 195

Joshi, Y. C. 2005, MNRAS, 362, 1259

Kim, Y.-C., Demarque, P., Yi, S. K., \& Alexander, D. R. 2002, ApJS, 143, 499

King, I. R. 1966, AJ, 71, 64

Koposov, S., de Jong, J. T. A., Belokurov, V., et al. 2007, ApJ, 669, 337

Kuchinski, L. E., \& Frogel, J. A. 1995, AJ, 110, 2844

Kuchinski, L. E., Frogel, J. A., Terndrup, D. M., \& Persson, S. E. 1995, AJ, 109, 1131

Küpper, A. H. W., Kroupa, P., Baumgardt, H., \& Heggie, D. C. 2010a, MNRAS, 407, 2241

Küpper, A. H. W., Kroupa, P., Baumgardt, H., \& Heggie, D. C. 2010b, MNRAS, 401, 105

Lamb, D. Q., Bulik, T., \& Coppi, P. S. 1996, in High Velocity Neutron Stars, ed. R. E. Rothschild, \& R. E. Lingenfelter, AIP Conf. Ser., 366, 219

Law, D. R., \& Majewski, S. R. 2010, ApJ, 718, 1128

Leitherer, C., Schaerer, D., Goldader, J. D., et al. 1999, ApJS, 123, 3

Longmore, A. J., Kurtev, R., Lucas, P. W., et al. 2011, MNRAS, 1184

López-Corredoira, M., Cabrera-Lavers, A., Garzón, F., \& Hammersley, P. L. 2002, A\&A, 394, 883

Mallen-Ornelas, G., \& Djorgovski, S. 1993, in Structure and Dynamics of Globular Clusters, ed. S. G. Djorgovski, \& G. Meylan, ASP Conf. Ser., 50, 313

Mermilliod, J. 1996, in The Origins, Evolution, and Destinies of Binary Stars in Clusters, ed. E. F. Milone, \& J.-C. Mermilliod, ASP Conf. Ser., 90, 475

Meylan, G., \& Heggie, D. C. 1997, A\&ARv, 8, 1

Minniti, D., Lucas, P. W., Emerson, J. P., et al. 2010, New A, 15, 433

Minniti, D., Hempel, M., Toledo, I., et al. 2011, A\&A, 527, A81

Monaco, L., Saviane, I., Correnti, M., Bonifacio, P., \& Geisler, D. 2011, A\&A, 525, A124

Moni Bidin, C., de La Fuente Marcos, R., de La Fuente Marcos, C., \& Carraro, G. 2010, A\&A, 510, A44

Nishiyama, S., Tamura, M., Hatano, H., et al. 2009, ApJ, 696, 1407

Origlia, L., Rich, R. M., \& Castro, S. M. 2001, in A\&AS Meeting Abstracts, BAAS, 33, 1386

Origlia, L., Valenti, E., \& Rich, R. M. 2005, MNRAS, 356, 1276

Ortolani, S., Barbuy, B., Bica, E., et al. 2001, A\&A, 376, 878

Ortolani, S., Bonatto, C., Bica, E., \& Barbuy, B. 2009, AJ, 138, 889

Pavani, D. B., \& Bica, E. 2007, A\&A, 468, 139

Peterson, C. J., \& Reed, B. C. 1987, PASP, 99, 20

Rieke, G. H., \& Lebofsky, M. J. 1985, ApJ, 288, 618

Roeser, S., Demleitner, M., \& Schilbach, E. 2010, AJ, 139, 2440

Rosenberg, A., Saviane, I., Piotto, G., Aparicio, A., \& Zaggia, S. R. 1998, AJ, 115,648

Saito, R., Hempel, M., Alonso-García, J., et al. 2010, The Messenger, 141, 24

Sakamoto, T., \& Hasegawa, T. 2006, ApJ, 653, L29

Salaris, M., \& Girardi, L. 2002, MNRAS, 337, 332

Salaris, M., Chieffi, A., \& Straniero, O. 1993, ApJ, 414, 580

Schechter, P. L., Mateo, M., \& Saha, A. 1993, PASP, 105, 1342

Schlegel, D. J., Finkbeiner, D. P., \& Davis, M. 1998, ApJ, 500, 525

Skrutskie, M. F., Cutri, R. M., Stiening, R., et al. 2006, AJ, 131, 1163

Sollima, A., Bellazzini, M., Smart, R. L., et al. 2009, MNRAS, 396, 2183

Stetson, P. B. 1994, PASP, 106, 250

Stetson, P. B., Hesser, J. E., Smith, G. H., Vandenberg, D. A., \& Bolte, M. 1989, AJ, 97, 1360

Valcarce, A. A. R. 2011, Ph.D. Thesis, Pontificia Universidad Católica de Chile

Valenti, E., Ferraro, F. R., \& Origlia, L. 2004a, MNRAS, 351, 1204

Valenti, E., Ferraro, F. R., Perina, S., \& Origlia, L. 2004b, A\&A, 419, 139

Valenti, E., Ferraro, F. R., \& Origlia, L. 2010, MNRAS, 402, 1729

van den Bergh, S., Morbey, C., \& Pazder, J. 1991, ApJ, 375, 594

VandenBerg, D. A., Swenson, F. J., Rogers, F. J., Iglesias, C. A., \& Alexander, D. R. 2000, ApJ, 532, 430

Villanova, S., Carraro, G., de la Fuente Marcos, R., \& Stagni, R. 2004, A\&A, 428, 67

Webbink, R. F. 1985, in Dynamics of Star Clusters, ed. J. Goodman, \& P. Hut, IAU Symp., 113, 541

Wilson, C. P. 1975, AJ, 80, 175

Zacharias, N., Monet, D. G., Levine, S. E., et al. 2004, in A\&AS Meeting Abstracts, BAAS, 36, 1418

Zacharias, N., Finch, C., Girard, T., et al. 2010, AJ, 139, 2184

Zoccali, M., Barbuy, B., Hill, V., et al. 2004, A\&A, 423, 507

\footnotetext{
${ }^{4}$ http://www . univie.ac .at/webda/
} 